\title{
Non-malleable Codes and Extractors for Small-Depth Circuits, and Affine Functions
}

\author{
Eshan Chattopadhyay \\ Institute for Advanced Study, Princeton \\ 1 Einstein Drive \\ Princeton, New Jersey 08540 \\ eshanc@ias.edu
}

\author{
Xin Li \\ Johns Hopkins University \\ Baltimore, Maryland 21218 \\ lixints@cs.jhu.edu
}

\begin{abstract}
Non-malleable codes were introduced by Dziembowski, Pietrzak and Wichs as an elegant relaxation of error correcting codes, where the motivation is to handle more general forms of tampering while still providing meaningful guarantees. This has led to many elegant constructions and applications in cryptography. However, most works so far only studied tampering in the split-state model where different parts of the codeword are tampered independently, and thus do not apply to many other natural classes of tampering functions. The only exceptions are the work of Agrawal et al, which studied non-malleable codes against bit permutation composed with bit-wise tampering, and the works of Faust et al and Ball et al, which studied non-malleable codes against local functions. However, in both cases each tampered bit only depends on a subset of input bits.

In this work, we study the problem of constructing non-malleable codes against more general tampering functions that act on the entire codeword. We give the first efficient constructions of nonmalleable codes against $\mathrm{AC}^{0}$ tampering functions and affine tampering functions. These are the first explicit non-malleable codes against tampering functions where each tampered bit can depend on all input bits. We also give efficient non-malleable codes against $t$-local functions for $t=o(\sqrt{n})$, where a $t$-local function has the property that any output bit depends on at most $t$ input bits. In the case of deterministic decoders, this improves upon the results of Ball et al, which can handle $t \leq n^{\frac{1}{4}}$.

All our results on non-malleable codes are obtained by using the connection between non-malleable codes and seedless nonmalleable extractors discovered by Cheraghchi and Guruswami. Therefore, we also give the first efficient constructions of seedless non-malleable extractors against $\mathrm{AC}^{0}$ tampering functions, $t$-local tampering functions for $t=o(\sqrt{n})$, and affine tampering functions. To derive our results on non-malleable codes, we design efficient algorithms to almost uniformly sample from the pre-image of any given output of our non-malleable extractor.
\end{abstract}

Permission to make digital or hard copies of all or part of this work for personal or classroom use is granted without fee provided that copies are not made or distributed for profit or commercial advantage and that copies bear this notice and the full citation on the first page. Copyrights for components of this work owned by others than ACM must be honored. Abstracting with credit is permitted. To copy otherwise, or republish, to post on servers or to redistribute to lists, requires prior specific permission and/or a fee. Request permissions from permissions@acm.org.

STOC'17, Montreal, Canada

(c) 2017 ACM. 978-1-4503-4528-6/17/06 ..\$15.00

DOI: $10.1145 / 3055399.3055483$

\section{CCS CONCEPTS}

- Mathematics of computing $\rightarrow$ Coding theory; • Security and privacy $\rightarrow$ Information-theoretic techniques; • Theory of computation $\rightarrow$ Pseudorandomness and derandomization;

\section{KEYWORDS}

Randomness Extractor, Pseudorandomness, Non-Malleable Extractors, Non-Malleable Codes

\section{ACM Reference format:}

Eshan Chattopadhyay and Xin Li. 2017. Non-malleable Codes and Extractors for Small-Depth Circuits, and Affine Functions. In Proceedings of 49th Annual ACM SIGACT Symposium on the Theory of Computing, Montreal, Canada, fune 2017 (STOC'17), 14 pages.

DOI: $10.1145 / 3055399.3055483$

\section{INTRODUCTION}

Error-correcting codes encode a message $m$ into a longer codeword $c$ enabling recovery of $m$ even after part of $c$ is corrupted. We can view this corruption as a tampering function $f$ acting on the codeword, where $f$ is from some small allowable family $\mathcal{F}$ of tampering functions. The strict requirement of retrieving the encoded message $m$ imposes restrictions on the kind of tampering functions that can be handled. Unique decoding is limited by the minimum distance of the code, and various bounds are known in the case of list decoding. Hence, many natural classes of tampering functions cannot be handled in this framework.

One might hope to achieve a weaker goal of only detecting errors, possibly with high probability. Cramer et al. [18] constructed one such class of error-detecting codes, known as Algebraic Manipulation Detection codes (AMD codes), where the allowable tampering functions consist of all functions of the form $f_{a}(x)=a+x$. However error detection is impossible with respect to the family of constant functions. This follows since one cannot hope to detect errors against a function that always outputs some fixed codeword.

Dziembowski, Pietrzak and Wichs [22] introduced non-malleable codes as a natural generalization of error-detecting codes. Informally, a non-malleable code with respect to a tampering function family $\mathcal{F}$ is equipped with a randomized encoder Enc and a deterministic decoder $\operatorname{Dec}$ such that $\operatorname{Dec}(\operatorname{Enc}(m))=m$ and for any tampering function $f \in \mathcal{F}$ the following holds: for any message $m$, $\operatorname{Dec}(f(\operatorname{Enc}(m)))$ is either the message $m$ or is $\epsilon$-close (in statistical distance) to a distribution $D_{f}$ independent of $m$. The parameter $\epsilon$ is called the error. Besides being a natural generalization of error correcting codes, [22] also showed that such non-malleable codes have several applications in tamper-resilient cryptography.

We now introduce some notions before formally defining nonmalleable codes. 
Definition 1.1. For any function $f: S \rightarrow S, f$ has a fixed point at $s \in S$ if $f(s)=s$. We say $f$ has no fixed points in $T \subseteq S$, if $f(t) \neq t$ for all $t \in T$. $f$ has no fixed points if $f(s) \neq s$ for all $s \in S$.

Definition 1.2 (TAMPERING FUNCTIONS). For any $n>0$, let $\mathcal{F}_{n}$ denote the set of all functions $f:\{0,1\}^{n} \rightarrow\{0,1\}^{n}$. Any subset of $\mathcal{F}_{n}$ is a family of tampering functions.

Further, a function is called $t$-local if every output bit depends on at most $t$ input bits.

Definition 1.3. The statistical distance between two distributions $\mathcal{D}_{1}$ and $\mathcal{D}_{2}$ over some universal set $\Omega$ is defined as $\left|\mathcal{D}_{1}-\mathcal{D}_{2}\right|=$ $\frac{1}{2} \sum_{d \in \Omega}\left|\operatorname{Pr}\left[\mathcal{D}_{1}=d\right]-\operatorname{Pr}\left[\mathcal{D}_{2}=d\right]\right|$. We say $\mathcal{D}_{1}$ is $\epsilon$-close to $\mathcal{D}_{2}$ if $\left|\mathcal{D}_{1}-\mathcal{D}_{2}\right| \leq \epsilon$ and denote it by $\mathcal{D}_{1} \approx_{\epsilon} \mathcal{D}_{2}$.

We now formally define non-malleable codes. We need to define the following function.

$$
\operatorname{copy}(x, y)= \begin{cases}x & \text { if } x \neq s a m e^{\star} \\ y & \text { if } x=s^{\star} a m e^{\star}\end{cases}
$$

$\operatorname{copy}^{(t)}\left(\left(x_{1}, \ldots, x_{t}\right),\left(y_{1}, \ldots, y_{t}\right)\right)=\left(\operatorname{copy}\left(x_{1}, y_{1}\right), \ldots, \operatorname{copy}\left(x_{t}, y_{t}\right)\right)$

Definition 1.4 (Coding Schemes). Let Enc $:\{0,1\}^{k} \rightarrow\{0,1\}^{n}$ and Dec $:\{0,1\}^{n} \rightarrow\{0,1\}^{k} \cup\{\perp\}$ be functions such that Enc is a randomized function (i.e., it has access to private randomness) and Dec is a deterministic function. We say that (Enc, Dec) is a coding scheme with block length $n$ and message length $k$ if for all $s \in\{0,1\}^{k}$, $\operatorname{Pr}[\operatorname{Dec}(E n c(s))=s]=1$ (the probability is over the randomness in Enc).

Definition 1.5 (Non-malleable codes). A coding scheme (Enc, Dec) with block length $n$ and message length $k$ is a non-malleable code with respect to a family of tampering functions $\mathcal{F} \subset \mathcal{F}_{n}$ and error $\epsilon$ if for every $f \in \mathcal{F}$ there exists a random variable $D_{f}$ on $\{0,1\}^{k} \cup\left\{\right.$ same $\left.e^{\star}\right\}$ which is independent of the randomness in Enc such that for all messages $s \in\{0,1\}^{k}$, it holds that

$$
\left|\operatorname{Dec}(f(\operatorname{Enc}(s)))-\operatorname{copy}\left(D_{f}, s\right)\right| \leq \epsilon .
$$

The rate of a non-malleable code $C$ is given by $\frac{k}{n}$.

As an easy example, suppose the tampering function family is $\mathcal{F}_{\text {constant }}$, consisting of all constant functions, $f_{c}(x)=c$ for all $x$. In this case, to get a non-malleable code we can use any coding scheme and for any tampering function $f_{c} \in \mathcal{F}_{\text {constant }}$, we can take $D_{f_{c}}$ to be $\operatorname{Dec}(c)$ with probability 1 .

Note that there cannot exist a code with block length $n$ which is non-malleable with respect to $\mathcal{F}_{n}$ (recall this is family of all functions from $n$ bits to $n$ bits). This follows since the tampering function could then use the function Dec to decode the message $m$, get a related message $m^{\prime}$ by flipping all the bits in $m$, and use the encoding function to pick any codeword in $\operatorname{Enc}\left(m^{\prime}\right)$.

Therefore, it is natural to restrict the size of the family of tampering functions. It follows from the works in $[12,22]$ that there exist non-malleable codes with respect to any tampering function family of size at most $2^{2^{\delta n}}$ with rate close to $1-\delta$ and error $2^{-\Omega(n)}$, for any constant $\delta>0$. The bounds obtained in these works are existential, and some progress has been made since then in giving explicit constructions against useful classes of tampering functions.
A well studied model of tampering functions is the $C$-split-state model, where we assume that the codeword is partitioned into $C$ parts and each part is independently tampered by an arbitrary function. Several works [1, 2, 7, 10, 12, 13, 20, 22, 29] studied this model resulting in explicit constructions of rate $\Omega(1 / \log n)$ nonmalleable codes in the 2 -split state model (note that there cannot exist non-malleable codes in the 1-split model).

However, a severe limitation of non-malleable codes in the splitstate model is that they cannot handle even simple tampering functions that depend on all bits of the codeword. In addition, very few work has constructed non-malleable codes for such tampering functions, partially because handling global functions seems challenging. Indeed, to the best of our knowledge, only the following works give non-malleable codes against a class of global tampering functions not captured by the split-state model in the information theoretic setting: the work of Faust et al. [23] gave efficient Monte-Carlo constructions against any family of tampering functions of size $2^{n^{C}}$. The work of Agrawal et al. [3], which studied non-malleable codes against bit permutation composed with bitwise tampering. In this case, the authors gave optimal constructions of non-malleable codes achieving rate $1-o(1)$ and error $2^{-\Omega(n)}$. The second one is a recent work by Ball et al. [5] , which constructed non-malleable codes against $t$-local functions with $t \leq n^{1 / 4}$ and rate $O\left(1 / t^{2}\right) .{ }^{1}$ We note that the class of tampering functions with bit permutation composed with bit-wise tampering is a special class of 1-local functions.

In this work, we make further progress towards constructing non-malleable codes that can handle more general global tampering functions. In particular, we give the first explicit construction of nonmalleable codes when the tampering functions are restricted to be in $\mathrm{AC}^{0}$ (constant depth circuits with unbounded fan-in gates). We also construct efficient non-malleable codes against $t$-local functions, with $t \leq n^{\frac{1}{2}-\delta}$ for any constant $0<\delta<1$. This improves the tolerance of locality of [5] in the case of deterministic decoders. Finally, we give the first explicit construction of non-malleable codes against affine tampering functions. Notice that the class of tampering functions which consists of bit permutation composed with bit-wise tampering is a strict subset of affine functions. Thus in terms of the class of tampering functions, our work subsumes that of [3], although we do not achieve optimal rate and error as in their construction. We also note that for $\mathrm{AC}^{0}$ tampering functions and affine tampering functions, each tampered bit can depend on all input bits. This is in contrast to all previous works where any tampered bit only depends on a subset of the input bits. Our results thus give the first explicit constructions of non-malleable codes against such tampering functions.

Our constructions of non-malleable codes exploit a particularly useful connection between such codes and seedless non-malleable extractors found by Cheraghchi and Guruswami [13].

We first recall the definition of seedless non-malleable extractors, which generalizes the more commonly studied seeded nonmalleable extractors [19].

\footnotetext{
${ }^{1}$ [5] also considers a relaxed notion of non-malleable codes where the decoder is allowed to be randomized as well, and shows how to handle locality up to $o(n / \log n)$ in this relaxed notion.
} 
Definition 1.6 ([14,35]). The min-entropy of a source $\mathrm{X}$ is defined to be: $H_{\infty}(\mathrm{X})=\min _{x}(-\log (\operatorname{Pr}[\mathrm{X}=x]))$. The min-entropy rate of $a$ source $\mathrm{X}$ on $\{0,1\}^{n}$ is $H_{\infty}(\mathrm{X}) / n$. Any source $\mathrm{X}$ on $\{0,1\}^{n}$ with minentropy at least $k$ is called an $(n, k)$-source.

We present a slightly simplified definition of seedless nonmalleable extractors, and refer the reader to Section 3.1 for the general definition.

Definition 1.7 (SeEdless NON-MALlEable eXtractors). Let $\mathcal{F} \subset \mathcal{F}_{n}$ be a family of tampering functions such that no function in $\mathcal{F}$ has any fixed points. A function nmExt : $\{0,1\}^{n} \rightarrow\{0,1\}^{m}$ is a seedless non-malleable extractor with respect to $\mathcal{F}$ and a class of sources $\mathcal{X}$ with error $\epsilon$ if for every distribution $\mathrm{X} \in \mathcal{X}$ and every tampering function $f \in \mathcal{F}$,

$\left|\operatorname{nmExt}(\mathbf{X}) \circ \operatorname{nmExt}(f(\mathbf{X}))-\mathbf{U}_{m} \circ \operatorname{nmExt}(f(\mathbf{X}))\right| \leq \epsilon$.

Further, we say that nmExt is $\epsilon^{\prime}$-invertible, if there exists an efficient sampling algorithm $\mathcal{A}$ that takes as input $y \in\{0,1\}^{m}$, and outputs a sample from a distribution that is $\epsilon^{\prime}$-close to the uniform distribution on the set $\mathrm{nmExt}^{-1}(y)$.

The only known constructions of seedless non-malleable extractors are in the case where the family $\mathcal{F}$ is the $C$-split-state tampering class $[7,10,29]$. In particular, when $C=2$, it amounts to constructing non-malleable extractors that have access to two independent sources $\mathbf{X}_{1}$ and $\mathbf{X}_{2}$, with $\mathbf{X}_{1}$ being independently tampered by a function $f_{1}$ and $\mathrm{X}_{2}$ being independently tampered by another function $f_{2}$.

In this work, we construct seedless non-malleable extractors that can handle functions which tamper the entire source globally. In particular, we give the first construction of seedless non-malleable extractors when the tampering functions are $\mathrm{AC}^{0}$ circuits. To the best of our knowledge, there was no known construction of seedless non-malleable extractors even when the tampering functions are from $\mathrm{NC}^{0}$. We also construct seedless non-malleable extractors for the class of $t$-local tampering functions and affine tampering functions. Using these constructions and the connection to nonmalleable codes by Cheraghchi and Guruswami [13], we obtain nonmalleable codes against the corresponding families of tampering functions.

\subsection{Our Results}

We state our results on non-malleable extractors assuming the tampering functions have no fixed points, since it is easier this way. However, all our results generalize to handle fixed points and we refer the reader to later sections of the paper for the more general versions of the theorems.

We first define oblivious bit-fixing sources and affine sources to present our results.

Definition 1.8. An oblivious bit-fixing source $\mathrm{X}$ on $n$ bits is a source where some subset of the bits are are chosen independently and uniformly and remaining bits are fixed (and do not depend on the choice of the random bits).

Definition 1.9. A distribution $\mathrm{X}$ on $\{0,1\}^{n}$ is called an affine source with min-entropy $k$ if it is uniform over some affine subspace in $\mathbb{F}_{2}^{n}$ of dimension $k$.
Our first result gives explicit seedless non-malleable extractors for affine sources against affine tampering functions. We note that no such construction was known even for the case of full entropy.

Theorem 1. For all $n, k>0$, any $\delta>0$ and $k \geq n-n^{\delta / 2}$, there exists an efficient function anmExt $:\{0,1\}^{n} \rightarrow\{0,1\}^{m}, m=n^{\Omega(1)}$, such that if $\mathrm{X}$ is an affine source with min-entropy at least $k$ and $\mathcal{A}:\{0,1\}^{n} \rightarrow\{0,1\}^{n}$ is an affine function with no fixed point, then $\left|\operatorname{anmExt}(\mathrm{X}), \operatorname{anmExt}(\mathcal{A}(\mathrm{X}))-\mathrm{U}_{m}, \operatorname{anmExt}(\mathcal{A}(\mathrm{X}))\right| \leq 2^{-n^{\Omega(1)}}$.

Next, we construct seedless non-malleable extractors for oblivious bit-fixing sources against $t$-local tampering functions. Again, we are not aware of any such explicit construction before (even for full entropy).

THEOREM 2. For any $\delta>0$ and all $n>0$, there exists an efficient function localnmExt : $\{0,1\}^{n} \rightarrow\{0,1\}^{m}, m=n^{\Omega(1)}$, such that if $\mathrm{X}$ is an oblivious bit-fixing source on $n$ bits with min-entropy $k$ and $f:\{0,1\}^{n} \rightarrow\{0,1\}^{n}$ is a $t$-local function, $t \leq k / n^{\frac{1}{2}+\delta}$ and has no fixed point, then

|localnmExt(X), localnmExt $(f(\mathbf{X}))-\mathbf{U}_{m}, \operatorname{localnmExt}(f(\mathbf{X})) \mid$

$$
\leq 2^{-n^{\Omega(1)}} \text {. }
$$

In particular, if we start with an oblivious bit-fixing source with min-entropy $k=\Omega(n)$, then we can handle $t$-local tampering functions for $t$ up to $n^{1 / 2-\delta}$.

Finally, we give seedless non-malleable extractors when the tampering functions are from $\mathrm{AC}^{0}$.

Theorem 3. For all $n>0$ and any $d=O(1)$, there exists an efficient function acnmExt : $\{0,1\}^{n} \rightarrow\{0,1\}^{m}, m=n^{\Omega(1)}$, such that if $\mathrm{X}$ is uniform on $n$ bits and $C:\{0,1\}^{n} \rightarrow\{0,1\}^{n}$ is an $\mathrm{AC}^{0}$ circuit of size at most $n^{d}$ with no fixed point, then

$\left|\operatorname{acnmExt}(\mathrm{X}), \operatorname{acnmExt}(C(\mathrm{X}))-\mathrm{U}_{m}, \operatorname{acnmExt}(C(\mathrm{X}))\right| \leq \frac{1}{n^{\Omega(\log n)}}$.

Next, we derive our results on non-malleable codes based on the above extractors. To use this connection, we need additional properties from the non-malleable extractors. Specifically, we need to be able to efficiently sample almost uniformly from the pre-image of any output. We show how to do this in Section 8, which is an essential ingredient for construting efficient non-malleable codes.

The following theorem gives efficient non-malleable codes against affine tampering. Prior to this, there were no known explicit constructions in this model.

THEOREM 4. There exists a constant $\gamma>0$ such that for all $n>0$ there exists an efficient construction of non-malleable codes against affine tampering functions with block-length $n$, relative rate $n^{\gamma} / n$ and error $2^{-n^{\Omega(1)}}$.

The following theorem gives efficient non-malleable codes against $t$-local tampering functions, allowing locality up to $t=n^{\frac{1}{2}-\delta}$.

Theorem 5. There exist constants $\gamma, \delta>0$ such that for all $n>0$ there exists an efficient construction of non-malleable codes against $t$-local tampering functions, $t \leq n^{\frac{1}{2}-\delta}$ with block-length $n$, relative rate $n^{\gamma} / n$ and error $2^{-n^{\Omega(1)}}$. 
Next, we give the first explicit constructions of non-malleable codes against the class of $\mathrm{AC}^{0}$ tampering functions.

THEOREM 6. There exists a constant $\gamma>0$ such that for all $n>0$ there exists an efficient construction of non-malleable codes for $\mathrm{AC}^{0}$ tampering functions with block-length $n$, relative rate $\left(\log ^{2} n\right) / n$ and error $n^{-\Omega(\log n)}$.

REMARK 1.10. Note that in our non-malleable codes for $\mathrm{AC}^{0}$ tampering functions, the block length is actually super-polynomial in the message length. However, the construction is still explicit and efficient in the sense that the encoding and decoding can be done in polynomial time of the block length. This notion of "efficient" is also generally used in previous constructions of non-malleable codes, such as that in [3].

\section{OVERVIEW OF THE CONSTRUCTIONS AND TECHNIQUES}

Here we provide an overview of our constructions and techniques. As mentioned earlier, our starting point is the general connection between non-malleable codes and seedless non-malleable extractors in [13], where Cheraghchi and Guruswami showed that nonmalleable extractors with sufficiently good parameters for a class of tampering functions implies non-malleable codes for such tampering functions. Therefore, we will first construct non-malleable extractors for $\mathrm{AC}^{0}$ tampering functions, local tampering functions and affine tampering functions, and then transform them into nonmalleable codes by providing efficient algorithms to sample almost uniformly from the pre-image of any output.

We deal with $\mathrm{AC}^{0}$ functions and local functions by an argument similar to that of Viola [34]. Specifically, a standard application of the switching lemma shows that if one applies a random restriction to an $\mathrm{AC}^{0}$ function, then with high probability the function collapses into a small depth decision tree. If the depth $d$ of the tree is small (e.g., $d<\log n$ ), then it is also a local function that depends on at most $2^{d}$ input bits. Thus we have reduced $\mathrm{AC}^{0}$ functions to local functions. Now, given any output bit $y_{i}$, by picking a particular input bit $x_{j}$ that $y_{i}$ depends on, and fixing all the neighbors of $\left\{y_{\ell}\right\}$ except $x_{j}$, where the set of $\left\{y_{\ell}\right\}$ consists of all neighbors of $x_{j}$ (here we view the input-output dependence as a bipartite graph), we can ensure that $y_{i}$ only depends on $x_{j}$. We repeat this process until each $y_{i}$ is either fixed or depends on a single $x_{j}$. In this way, the function now becomes an affine function on the input bits. We note that an important difference between our case and the case of Viola [34] is that, there one needs to preserve the min-entropy of the output bits (since the goal is to design an extractor for the output source), while here we don't need that. All we need is that the function now becomes an affine function, while some of the input bits are left unfixed. In other words, the initial input bits now become an oblivious bit-fixing source. Thus, we have reduced the problem of constructing non-malleable extractors with respect to $\mathrm{AC}^{0}$ and local tampering functions to the problem of constructing non-malleable extractors for bit-fixing sources with respect to affine tampering functions (in fact, a very special class of affine tampering functions where each output bit depends on at most one input bit).

\subsection{The Non-malleable Extractor}

For simplicity, let us assume that the tampering function has no fixed point (we show in Section 3.1 that such non-malleable extractors also imply general non-malleable extractors that can deal with fixed points). Now our high level idea of constructing the non-malleable extractor will follow the non-malleable two-source extractor of [7], in which one first obtains a small advice and then use a correlation breaker with advice (implicitly introduced in [7] and formally defined in [17]) to get the final output. One can show that with high probability the advice obtained from the initial (untampered) sources is different from the advice obtained from the tampered sources. Thus the correlation breaker with advice guarantees that the output of the extractor is uniform given the tampered output.

Here we would like to do something similar. However, there are several tricky issues that we need to solve. First, how do we generate the advice? Second, once we have the advice, how do we construct the correlation breaker? Our starting point is the correlation breaker for affine sources developed in [9,27], which is based on alternating extraction between part of the affine source and the source itself, using strong linear seeded extractors. Assuming that we have already obtained the advice (which is different from its tampered version), and conditioned on the fixing of the advice and its tampered version, the original source is still an affine source, then we can use the above described correlation breaker to obtain an output that is uniform given its tampered version. Yet, this leads to another problem: to use the affine correlation breaker, we need the original affine source to have high entropy, while here (after the random restriction and fixing additional bits) the source can have quite small entropy.

Luckily, what we have here is a special kind of affine source an oblivious bit-fixing source. For such sources we can first use the linear condenser developed in [30,34] to get another small affine source which has some entropy. The point is that this new affine source has a small length, which is smaller than the entropy of the original bit-fixing source. Thus we can apply a strong linear seeded extractor to convert the new source into a somewhere random matrix by trying all possible seeds (a somewhere random matrix is a random matrix such that at least one row is uniform.) and then use each row of the matrix to extract from the original source (by using another strong linear seeded extractor). The property of strong linear seeded extractors guarantees that we end up with another somewhere random matrix which has a small number of rows where each row is quite long. In addition, conditioned on the fixing of the short affine source, each row of the new matrix is a linear function of the original source. The new matrix is much easier to handle, and will be the starting point of our following constructions.

The somewhere random matrix also makes it easier for us to obtain the advice. Specifically, the advice in [7] is obtained by taking a small slice of each source (recall that there we have two independent high min-entropy source), and use a function of the two small slices (actually, a two-source extractor such as inner product) to sample some bits from the encoding of each source by an error correcting code. The final advice is the concatenation of the two slices and the sampled bits. The analysis is that if the slices are different from their tampered version in the first place, 
then we are already done; otherwise with high probability the sampled bits are different from their tampered version. Here, since we have a somewhere random source, if we know which row is uniform, then we can try to take a small slice of that row and do something similar. In order to keep the source to be an affine source given the fixing of the advice, we will need to use a linear error correcting code. However, again we have two problems. First, we don't know which row is the uniform row. Thus, we will use each row to produce an advice, and we append the index of each row to the corresponding advice to guarantee that the advice from the "good" row is different from all the other advices (including the tampered advices and the advices from other rows). Since the number of rows is small compared to the length of each row, we can fix all the advices and each row still has high entropy. Now we can apply the correlation breaker to each row and the original source X, and finally take the XOR of the outputs, which is still enough for our purpose. Second, and more seriously, unlike the setting of [7] where we have two independent sources, now we only have one affine source $\mathrm{X}$. This means that each row in the somewhere random matrix is actually correlated with the original source $\mathbf{X}$. Therefore it is not clear if the sampling from an encoding of $\mathrm{X}$ can give us anything, since the random bits used to sample from the encoding are actually correlated with $\mathrm{X}$. It is indeed quite non-trivial to make this work, but given that the source is an affine source and the tampering function is also affine, we managed to show (in Section 5.3) that an appropriate modification of the advice generator in [7] still works in this case. This gives the construction of our non-malleable extractor.

We note that the above construction works not only for bit-fixing sources, but also for any low-weight affine sources ${ }^{2}$. In addition, it also works for general affine sources with high entropy, since in this case we can just take a small slice to serve the purpose of what we get from the linear condenser.

\subsection{Efficient Sampling}

We now turn to our algorithm to efficiently sample almost uniformly form the pre-image of any given output. Since our construction uses the affine correlation breaker which consists of multiple steps of alternating extraction, to directly inverting this process such as that done in [7] seems pretty troublesome. Thus, we would like to use the much simpler sampling method recently developed by $\mathrm{Li}$ [29], which treats most of the construction details as a black box. A direct translation of that method to our case results in the following modified extractor construction and sampling algorithm: first, we modify the last step of the extractor construction and apply the correlation breaker to two new larger slices of each row (or a slice of each row and the concatenation of another slice from every row). When we have obtained the outputs from the correlation breaker, we use each of them to extract from a new, longer part of the corresponding row using a linear invertible seeded extractor developed in $[7,29]$. Note here we are using longer and longer slices from each row. The correlation breaker and the linear invertible seeded

${ }^{2} \mathrm{~A}$ low-weight affine source is the uniform distribution over some affine subspace which can be expressed as the linear combination of vectors with low Hamming weight.

extractor both require their input source to have high entropy (conditioned on the fixing of previous random variables), which can be guaranteed since the length of each row is much larger than the number of rows in the somewhere random matrix. Finally, we take the XOR of all the outputs.

Now to sample from the pre-image of a given output, we first uniformly generate the small affine source $\mathrm{V}$ which we obtain from the linear condenser. This gives us a system of linear equations on the bits of the original source $\mathrm{X}$ (obtained from $\mathrm{V}$ ), and another system of linear functions of the bits of $\mathrm{X}$ corresponding to each row in the somewhere random matrix. Then, we uniformly generate the slice of each row and the advices, which give us a system of linear equations. We now uniformly generate a larger slice of each row and apply the correlation breaker to obtain the outputs. Finally, given the output of the non-malleable extractor we uniformly generate each part that appears in the XOR of the outputs of the linear invertible seeded extractor, and use the invertible extractor to obtain the pre-images which correspond to the parts of the rows in the somewhere random matrix that are used as the inputs to the linear invertible extractor. Thus we have obtained the somewhere random matrix, now we put together all the linear equations we have obtained before and we uniformly generate $\mathrm{X}$ according to these equations.

A serious problem with the above approach is that each time we obtain a system of linear equations, the rank of the coefficient matrix may not be the same. Thus if we just naively solve the equations and uniformly generate the pre-image, then overall the distribution may not be the uniform distribution over the pre-image of a given output. To solve this problem, we need to find a way to ensure that almost surely the coefficient matrix has the same rank. Again, this is quite non-trivial to achieve, and indeed most of our effort in the sampling part goes into ensuring this property.

We illustrate our ideas by staring from the first steps of the extractor construction. When we use the linear condenser to obtain the short affine source $V$, we create a system of linear equations between the bits of $\mathrm{X}$ and the bits of $\mathrm{V}$. Note that the coefficient matrix of this system is fixed. Next we use $\mathbf{V}$ and a strong linear seeded extractor to convert $X$ into a somewhere random matrix $\mathbf{R}$, where conditioned on the fixing of $\mathrm{V}$ each row is a linear function of $\mathbf{X}$. Here however, for different fixing of $\mathbf{V}=v$ the coefficient matrix is different and may have different rank. In addition, it may have different dependence on the previous system of equations (which is obtained from V). Our solution to this problem is that we are going to manually pick a subset of the rows from the matrix $\mathbf{R}$, and for each row in the subset we manually pick a subset of its bits. We will ensure that by doing this, the new matrix is still a somewhere random matrix, but with the additional property that the linear functions given by the bits in this matrix are linearly independent, and further they are linearly independent of the previous system of equations (obtained from $\mathrm{V}$ ).

The idea is as follows. First, we can show by a standard argument that with high probability $\left(1-2^{-n^{\Omega(1)}}\right)$ over the fixing of $\mathbf{V}$, most of the rows (i.e., $1-2^{-n^{\Omega(1)}}$ fraction) in the somewhere random matrix $\mathbf{R}$ are uniform. Assuming this happens, now our crucial observation is that, (1) if the output bits are uniform, then the linear functions corresponding to these bits must be linearly independent, and (2) linear independence of vectors can be tested efficiently. Indeed, if 
the linear functions corresponding to some bits are linearly dependent, then these bits already have a linear correlation and cannot be uniform. Therefore, assuming that we have $D$ rows in the matrix $\mathbf{R}$, then we know that there are at least $\left(1-2^{-n^{\Omega(1)}}\right) D$ rows such that if we look at each row, then the linear functions corresponding to the bits in the row are linearly independent. We can thus pick say $0.9 D$ such rows. Note that this also guarantees that at least one row is still uniform, since we throw away only $0.1 D$ rows. However, the bits in different rows might be correlated, and may also have correlation with the system of equations obtained from $\mathrm{V}$. Thus, our next step is to go through the $0.9 D$ rows one by one, and for each row select some $\ell$ bits. We select these bits in such a way that they are linearly independent of the equations obtained from $\mathrm{V}$, and the bits selected later are linearly independent of the bits selected before. We can do this because the bits in each row are linearly independent, and thus they span a subspace of dimension $m$ if $m$ is the length of each row in the matrix R. Suppose the length of $\mathrm{V}$ is $s$. Then as long as $m>s+D \ell$, we know that when we look at any particular row, there will always be bits that are linearly independent of the previous bits and the equations obtained from $\mathrm{V}$ (otherwise the subspace spanned by the bits in this row will have dimension at most $s+0.9 D \ell$ ). Finally, note that if a row is uniform, then any subset of its bits are also uniform. Therefore at the end we obtain a new somewhere random matrix $\mathbf{R}^{\prime}$, whose coefficient matrix of the bits has a fixed rank and is linearly independent of the equations obtained from $\mathbf{V}$.

The above is the basic idea behind our approach to ensure the coefficient matrix of the final system of linear equations has the same rank. We then carry it out in the following steps of the extractor construction. In the next step where we generate the advices, two additional problems arise. First, we need to use a slice of each row of $\mathbf{R}^{\prime}$ to sample from the encoding of the original source $\mathrm{X}$ by some linear error correcting code. We would like to make sure that the sampled bits have the same rank and are linearly independent of the bits in $\mathbf{V}$ and the used slices. This however is hard to guarantee if we sample some bits for each row. Thus, we modify the construction again to use the concatenation of the slices from each row (which is now a weak random source) to sample a single string from the encoding of $\mathrm{X}$. It is well known how to use weak random sources to do sampling (e.g., by using a seeded extractor). To ensure linearly independence, we use a dual $\mathrm{BCH}$ code, $\mathrm{dBCH}$, to encode $\mathrm{X}$ with the parameter $t_{\mathrm{BCH}}$ set such that $\mathrm{dBCH}$ has constant distance and polynomial rate. Thus the corresponding $\mathrm{BCH}$ code has minimum distance at least $2 t$. By a well known fact from coding theory, it thus guarantees that any $2 t_{\mathrm{BCH}}$ bits in the codeword are linearly independent. Next, we throw away those bits that may be dependent of the bits in $\mathbf{V}$ and the used slices. We choose the parameters such that the number of bits from $\mathbf{V}$ and the used slices is at most $n^{\alpha}$ for some small constant $0<\alpha<1$, thus we can sample $n^{\beta}$ coordinates from the $\mathrm{dBCH}$-encoding of $\mathrm{X}$ with $\beta>\alpha$ and $n^{\beta}<<t$. Since the $\mathrm{dBCH}$-code has a constant relative distance, we can show with high probability that the number of different bits (from the tampered version) in the sampled bits is a constant fraction. Thus even if we throw away $o(1)$ fraction of the sampled bits, we are still left with many different bits. When doing this, we also make sure the number of remaining sampled bits is the same, thus they have the same rank.
Second, the advice generating process is actually quite subtle due to the correlated sampling problem we described above; and in fact we need to use another random variable which is uniform conditioned on the fixing of the slices to extract from $\mathrm{X}$, to get another part of the advice. We do this by using a new, larger slice and use a linear seeded extractor with all possible seeds to convert it to a somewhere random matrix first, and then use each row to extract from $\mathrm{X}$ and concatenate all the outputs. When doing this, again we need to use the above described process to select from the output bits those that are linearly independent and independent of previously obtained bits from $\mathrm{V}$, the used slices and the previous advice.

After we are done with the above step, we actually introduced some new linear constraints which may be dependent of the remaining bits in each row of $\mathbf{R}^{\prime}$ (note that we cannot ensure the bits we obtained from the advices are linearly independent of all the bits in the somewhere random matrix $\mathbf{R}^{\prime}$, since we need the size of the advices to be smaller than the length of each row in $\mathbf{R}^{\prime}$, so that even conditioned on the fixing of the advices the good row in $\mathbf{R}^{\prime}$ still has high min-entropy). Thus, we repeat the picking process above to pick from the remaining bits of $\mathbf{R}^{\prime}$ those that are linearly independent and independent of previously obtained bits. As long as each row of $\mathbf{R}^{\prime}$ is long enough, we can always succeed in this step. Finally we can compute the output of the correlation breakers, and use the linear invertible extractor to generate the remaining bits of $\mathbf{R}^{\prime}$. Eventually, when we put them together we can argue that almost surely (with probability $1-2^{-n^{\Omega(1)}}$ ) the final system of linear equations has the same rank. Thus we can almost uniformly sample from the pre-image of any given output.

\section{SEEDLESS NON-MALLEABLE EXTRACTORS FOR LOW-WEIGHT AFFINE SOURCES AGAINST AFFINE ADVERSARIES}

We begin by defining a sub-class of affine sources, called low-weight affine sources, which was first studied by Rao [30].

Definition 3.1 (Low-Weight Affine Source). Any affine source $\mathrm{X}$ with min-entropy $k$ which has a set of basis vectors $\left\{v_{1}, \ldots, v_{k}\right\}$ such that the hamming weight of each $v_{i}$ is bounded by $w$ is called a $w$-affine source.

Rao [30] constructed extractors for $k^{\epsilon}$-affine sources for minentropy $k \geq \log ^{C} n$, for some constant $c$. This was subsequently improved by Viola [34] to achieve explicit extractors for $k^{1-\delta}$-affine sources.

We construct explicit seedless non-malleable extractors for $k^{1-\delta}$ affine sources against arbitrary affine tampering functions.

\subsection{Tampering Functions with Fixed Points}

Here we show that in the cases that are relevant to our applications, our non-malleable affine extractors for tampering functions with no fixed points imply general non-malleable affine extractors for arbitrary tampering functions. We refer the reader to the full version for proofs of the results stated in this section. We first recall the definition of a general seedless non-malleable extractor w.r.t. a class of tampering functions. 
Definition 3.2 (SeEdless Non-Malleable Extractor). A function nmExt $:\{0,1\}^{n} \rightarrow\{0,1\}^{m}$ is a $(k, \varepsilon)$-seedless non-malleable extractor with respect to a class $\mathcal{X}$ of sources over $\{0,1\}^{n}$ and a class $\mathcal{F}$ of tampering functions acting on $\{0,1\}^{n}$, if for every $\mathrm{X} \in \mathcal{X}$ with min-entropy $k$ and every $f \in \mathcal{F}$, there is a distribution $\mathcal{D}$ over $\{0,1\}^{m} \cup\left\{\right.$ same $\left.e^{\star}\right\}$ such that for an independent $\mathrm{Y}$ sampled from $D$, we have

$$
(\operatorname{nmExt}(\mathrm{X}), \operatorname{nmExt}(f(\mathrm{X}))) \approx_{\varepsilon}\left(U_{m}, \operatorname{copy}\left(\mathrm{Y}, U_{m}\right)\right),
$$

where the second $U_{m}$ is the same random variable as the first one.

We define the following two classes of tampering functions. Let $\mathcal{F}_{\text {affine }}$ be the set of tampering functions from $\{0,1\}^{n}$ to $\{0,1\}^{n}$ where each output bit is an affine function of the input bits. Let $\mathcal{F}_{\text {baffine }}$ be a subset of $\mathcal{F}_{\text {affine }}$, where for each $f \in \mathcal{F}_{\text {baffine }}$ and every $i \in[n], f(x)_{i}$ is either $x_{j}$ or $x_{j} \oplus 1$ for some $j \in[n]$, or a fixed bit. We show the following two lemmas.

LEMMA 3.1. Let nmExt be $a(k-2 k / w, \varepsilon)$-non-malleable extractor for weight $w$ affine sources, w.r.t affine tampering functions with no fixed points. Then nmExt is a $\left(k, \varepsilon+(n+1) 2^{-k / w}\right)$-non malleable extractor for oblivious bit-fixing sources, w.r.t. $\mathcal{F}_{\text {baffine }}$.

Our second lemma is in a similar flavor.

Lemma 3.2. Let nmExt be $a(k-\ell, \varepsilon)$-non-malleable extractor for affine sources, w.r.t affine tampering functions with no fixed points. Then nmExt is a $\left(k, \varepsilon+(n+1) 2^{-\ell}\right)$-non malleable extractor for affine sources w.r.t. $\mathcal{F}_{\text {affine }}$.

\section{PRELIMINARIES}

\subsection{Explicit Extractors from Prior Work}

We recall an optimal construction of strong-seeded extractors.

Theorem 4.1 ([24]). For any constant $\alpha>0$, and all integers $n, k>0$ there exists a polynomial time computable strong-seeded extractor Ext : $\{0,1\}^{n} \times\{0,1\}^{d} \rightarrow\{0,1\}^{m}$ with $d=O(\log n+$ $\log (1 / \epsilon))$ and $m=(1-\alpha) k$.

The following is an explicit construction of linear seeded extractors.

Theorem $4.2([31,32])$. For every $n, k, m \in \mathbb{N}$ and $\epsilon>0$, with $m \leq k \leq n$, there exists an explicit strong linear seeded extractor LExt : $\{0,1\}^{n} \times\{0,1\}^{d} \rightarrow\{0,1\}^{m}$ for min-entropy $k$ and error $\epsilon$, where $d=O\left(\frac{\log ^{2}(n / \epsilon)}{\log (k / m)}\right)$.

THEOREM 4.3. There exists a constant $\alpha$ such that for all $n>0$, and $\epsilon>2^{-\alpha n}$, there exists a strong linear seeded extractor iExt satisfying the following: If $X$ is a $(n, 0.9 n)$ source and $S$ is an independent uniform seed on $\{0,1\}^{d}, d=O(\log (n / \epsilon))$, then the following holds:

$$
\left|i \operatorname{Ext}(X, S), S-U_{m}, S\right|<2^{-n^{\Omega(1)}},
$$

where $m=\Omega(d)$. Further for any $r \in\{0,1\}^{m}$ and any $s \in\{0,1\}^{d}$, $\left|\mathrm{iExt}(\cdot, s)^{-1}(r)\right|=2^{n-m}$.

We also use a seeded extractor construction by Zuckerman [37] that achieves seed length $\log (n)+O\left(\log \left(\frac{1}{\epsilon}\right)\right)$ to extract from any source with constant min-entropy rate.

Theorem 4.4 ([37]). For all $n>0$ and constants $\alpha, \delta, \epsilon>0$ there exists an efficient construction of $a(k=\delta n, \epsilon)$-strong seeded extractor Ext : $\{0,1\}^{n} \times\{0,1\}^{d} \rightarrow\{0,1\}^{m}$ with $m \geq(1-\alpha) k$ and $D=2^{d}=O(n)$. [30].

We use a property of linear seeded extractors proved by Rao

Lemma 4.1 ([30]). Let Ext $:\{0,1\}^{n} \times\{0,1\}^{d} \rightarrow\{0,1\}^{m}$ be a linear seeded extractor for min-entropy $k$ with error $\epsilon<\frac{1}{2}$. Let $X$ be an affine $(n, k)$-source. Then

$$
\operatorname{Pr}_{u \sim U_{d}}\left[\left|\operatorname{Ext}(X, u)-U_{m}\right|>0\right] \leq 2 \epsilon .
$$
[6]

We recall an explicit affine extractor constructed by Bourgain

TheOREM 4.5. For all $n, k>0$ and any constant $\delta>0$ there exists an explicit affine extractor aExt : $\{0,1\}^{n} \rightarrow\{0,1\}^{m}, m=\Omega(k)$, for min-entropy $k$ with error $2^{-\Omega(k)}$.

\subsection{Sampling Using Weak Sources}

Seeded extractors can be used as samplers with access to weak sources. Recall a graph-theoretic view of seeded extractors. A seeded extractor Ext : $\{0,1\}^{n} \times\{0,1\}^{d} \rightarrow\{0,1\}^{m}$ can be viewed as an unbalanced bipartite graph $G_{\text {Ext }}$ with $2^{n}$ left vertices (each of degree $2^{d}$ ) and $2^{m}$ right vertices. Let $\mathcal{N}(x)$ denote the set of neighbors of $x$ in $G_{\text {Ext }}$.

Theorem $4.6([36])$. Let Ext $:\{0,1\}^{n} \times\{0,1\}^{d} \rightarrow\{0,1\}^{m}$ be a seeded extractor for min-entropy $k$ and error $\epsilon$. Let $D=2^{d}$. Then for any set $R \subseteq\{0,1\}^{m}$,

$$
\left|\left\{x \in\{0,1\}^{n}:|| \mathcal{N}(x) \cap R\left|-\mu_{R} D\right|>\epsilon D\right\}\right|<2^{k},
$$

where $\mu_{R}=|R| / 2^{m}$.

Theorem 4.7 ([36]). Let Ext : $\{0,1\}^{n} \times\{0,1\}^{d} \rightarrow\{0,1\}^{m}$ be a seeded extractor for min-entropy $k$ and error $\epsilon$. Let $\{0,1\}^{d}=\left\{r_{1}, \ldots\right.$, $\left.r_{D}\right\}, D=2^{d}$. Define $\operatorname{Samp}(x)=\left\{\operatorname{Ext}\left(x, r_{1}\right), \ldots, \operatorname{Ext}\left(x, r_{D}\right)\right\}$. Let $\mathrm{X}$ be an $(n, 2 k)$-source. Then for any set $R \subseteq\{0,1\}^{m}$,

$$
\operatorname{Pr}_{\mathbf{X} \sim \mathbf{X}}\left[|| \operatorname{Samp}(\mathbf{x}) \cap R\left|-\mu_{R} D\right|>\epsilon D\right]<2^{-k},
$$
where $\mu_{R}=|R| / 2^{m}$.

\section{NON-MALLEABLE CODES VIA SEEDLESS NON-MALLEABLE EXTRACTORS}

We recall a connection discovered between non-malleable codes and seedless non-malleable extractors by Sheraghchi and Guruswami [13]. The version we state here is more general than stated in [13]. It is easy to see that their proof generalizes to this more general version.

Theorem 5.1. Let nmExt $:\{0,1\}^{n} \rightarrow\{0,1\}^{m}$ be a polynomial time computable seedless non-malleable extractor that works for minentropy $n$ with error $\epsilon$ with respect to a class of tampering functions $\mathcal{F}$ acting on $\{0,1\}^{n}$. Further suppose there is a sampling algorithm Samp that on any input $z \in\{0,1\}^{m}$ runs in time poly $(n)$ and samples from a distribution that is $\epsilon^{\prime}$-close to uniform on the set $\mathrm{nmExt}^{-1}(s)$.

Then there exists an efficient construction of a non-malleable code with respect to the tampering family $\mathcal{F}$ with block length $=n$, relative rate $\frac{m}{n}$ and error $2^{m} \epsilon+\epsilon^{\prime}$. 

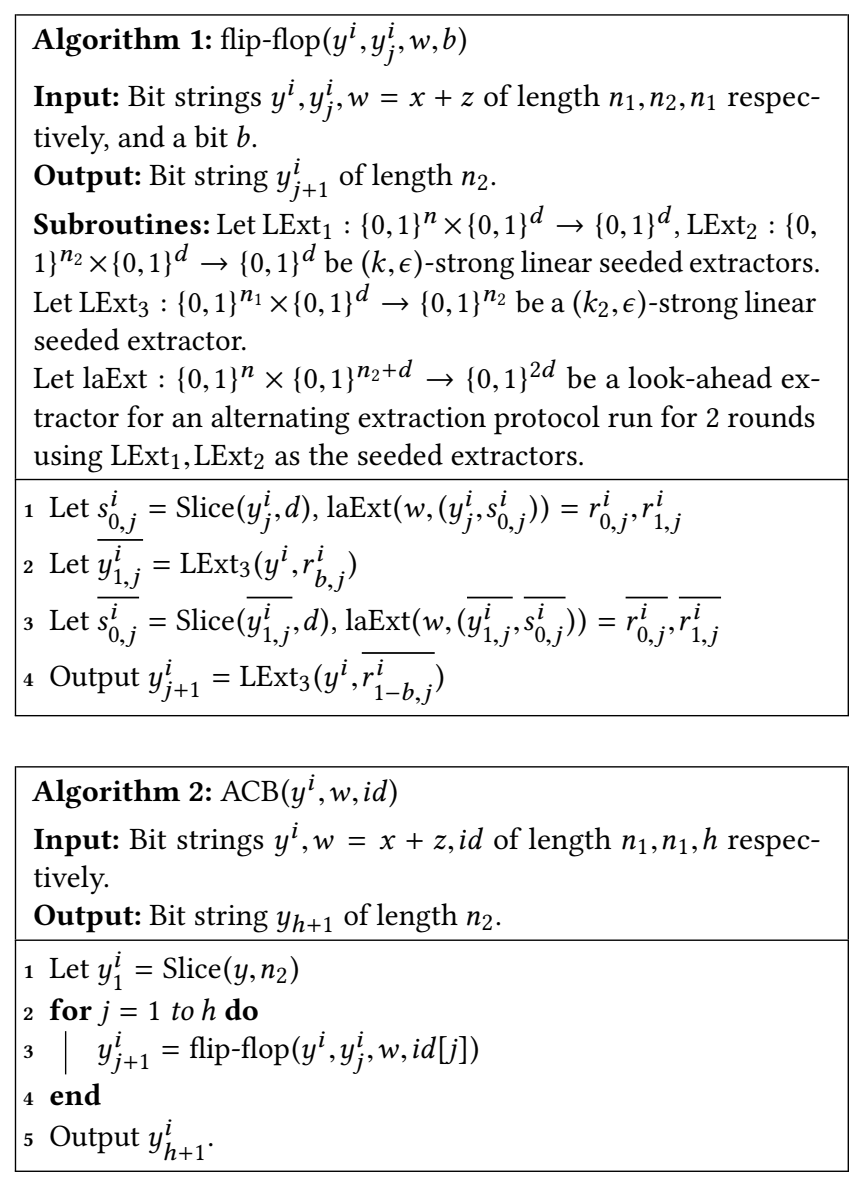

The non-malleable code is define in the following way: For any message $s \in\{0,1\}^{m}$, the encoder of the non-malleable code outputs Samp $(s)$. For any codeword $c \in\{0,1\}^{n}$, the decoder outputs $\operatorname{nmExt}(c)$.

\subsection{A Linear Condenser}

We use a linear condenser for low-weight affine sources [30, 34]. The condenser is essentially the parity check matrix of a binary code with high relative rate that achieves good relative distance as well (see e.g., [4]).

LEMMA 5.1 ([30, 34]). For any constant $0<\alpha<1$, and for all $n, k, w \in \mathbb{N}$, there exists a linear function LCon : $\{0,1\}^{n} \rightarrow\{0,1\}^{n_{1}}$, $n_{1}=O\left(w k^{\alpha} \log n\right)$ such that if $\mathrm{X}$ is a $w$-affine source with minentropy $k$, then $\operatorname{LCon}(X)$ has min-entropy at least $k^{\alpha}$.

\subsection{Some Primitives from Prior Work}

Let $\mathrm{Y}^{1}, \ldots, \mathrm{Y}^{t}$ be correlated r.v's. We recall an explicit construction from [9], that breaks the correlations between these r.v's using an additional correlated source of the form $\mathrm{X}+\mathrm{Z}$, assuming $\mathrm{X}$ is independent of $\mathrm{Z}, \mathrm{Y}^{1}, \ldots, \mathrm{Y}^{t}$ (and $\mathrm{Z}$ is allowed to have arbitrary correlations with $\mathrm{Y}^{1}, \ldots, \mathrm{Y}^{t}$ ). The technique is based on the powerful method of alternating extraction, which was introduced by Dziembowski and Pietrzak [21] and has found many applications in many explicit constructions of pseudorandom objects (e.g., [7, 9, 15, 19, 2628]).

Alternating Extraction We briefly recall the method of alternating extraction. Assume that there are two parties, Quentin with a source $Q$ and a uniform seed $S_{0}$, and Wendy with a source $W$. The alternating extraction protocol is an interactive process between Quentin and Wendy, and starts off with Quentin sending the seed $\mathrm{S}_{0}$ to Wendy. Wendy uses $\mathrm{S}_{0}$ and a strong seeded extractor Ext ${ }_{w}$ to extract a seed $\mathbf{R}_{0}=\operatorname{Ext}_{w}\left(\mathbf{W}, \mathrm{S}_{0}\right)$ using $\mathbf{W}$, and sends $\mathbf{R}_{0}$ back to Quentin. This constitutes a round of the alternating extraction protocol. In the next round, Quentin uses a strong extractor Ext $_{q}$ to extract a seed $\mathrm{S}_{1}=\operatorname{Ext}_{q}\left(\mathrm{Q}, \mathbf{R}_{o}\right)$ from $\mathrm{Q}$ using $\mathbf{R}_{0}$, and sends it to Wendy and so on. The protocol is run for $h$ steps, where $h$ is an input parameter. Thus, the following sequence of random variables is generated:

$$
\begin{array}{r}
\mathrm{S}_{0}, \mathrm{R}_{0}=\operatorname{Ext}_{w}\left(\mathbf{W}, \mathrm{S}_{0}\right), \mathrm{S}_{1}=\operatorname{Ext}_{q}\left(\mathrm{Q}, \mathrm{R}_{0}\right), \ldots, \\
\mathrm{S}_{h}=\operatorname{Ext}_{q}\left(\mathrm{Q}, \mathrm{R}_{h-1}\right), \mathbf{R}_{h}=\operatorname{Ext}_{w}\left(\mathbf{W}, \mathrm{S}_{h}\right) .
\end{array}
$$

Look-Ahead Extractor: We define the following look-ahead extractor:

$$
\operatorname{laExt}\left(\mathbf{W},\left(\mathbf{Q}, \mathrm{S}_{0}\right)\right)=\mathbf{R}_{1}, \ldots, \mathbf{R}_{h} .
$$

Algorithm 1 uses alternating extraction in a flip-flop way. This was introduced by Cohen [15], and has been extensively used in explicit constructions of pseudorandom objects [7-9, 15-17].

Algorithm 2 chains together several flip-flop steps along with an 'advice' string. This object is called a correlation breaker with advice, and was implicitly introduced by Chattopadhyay et al. [7]. This has since been used in other constructions $[9,17]$.

The following result was proved by Chattopadhyay and Li [9]. We state here a version that slightly more general. Since this is easy to obtain from the original proof in [9], we do not repeat the proof but briefly sketch the ideas to get this more general theorem. We allow the seed $\mathrm{Y}^{1}$ to be a $\left(n_{1}, n_{1}-\lambda\right)$-source (instead of being fully uniform). Using the fact that any strong seeded extractor with error $\epsilon$ also works for a weak seed with deficiency $\lambda$ with error $2^{\lambda} \epsilon$ (e.g., see Lemma 6.4 in [7]), the error parameter in Theorem 5.2 changes appropriately. Further, we allow tamperings on the sources $\mathrm{X}$ and $\mathbf{Z}$ as well, and it is easy to see that the proof in [9] (which is based on alternating extraction) generalizes to handle this as well.

TheOREM 5.2. For all integers $n, n_{1}, n_{2}, k, k_{1}, k_{2}, t, d, h, \lambda$ and any $\epsilon>0$, such that $k_{1} \geq k+8 t d h+\log (1 / \epsilon), n_{1} \geq k+10 t d h+(4 h t+$ 1) $n_{2}^{2}+\log (1 / \epsilon), n_{2} \geq k+3 t d+\log (1 / \epsilon)$ and $k_{2} \geq n_{2}^{1.1}$, let

- $\mathrm{X}$ be an $\left(n, k_{1}\right)$-source, $\mathrm{X}^{\prime}$ a r.v on $n$ bits, $\mathrm{Y}^{1}$ be an $\left(n_{1}, n_{1}-\lambda\right)$ source, $\mathrm{Z}, \mathrm{Z}^{\prime}$ are r.v's on $n$ bits, and $\mathrm{Y}^{2}, \ldots, \mathrm{Y}^{t}$ be r.v's on $n_{1}$ bits each, such that $\left\{\mathrm{X}, \mathrm{X}^{\prime}\right\}$ is independent of $\left\{\mathbf{Z}, \mathbf{Z}^{\prime}, \mathrm{Y}^{1}, \ldots\right.$, $\left.\mathrm{Y}^{t}\right\}$.

- $i d^{1}, \ldots, i d^{t}$ be bit-strings of length $h$ such that for each $i \in$ $\{2, t\}, i d^{1} \neq i d^{i}$.

- $\mathrm{Y}_{h+1}^{1}=\mathrm{ACB}\left(\mathrm{Y}^{1}, \mathrm{X}+\mathrm{Z}, i^{i}\right)$ where $\mathrm{ACB}$ is the function computed by Algorithm 2.

- $\mathrm{Y}_{h+1}^{i}=\mathrm{ACB}\left(\mathrm{Y}^{i}, \mathrm{X}^{\prime}+\mathrm{Z}^{\prime}, i d^{i}\right), i \in[2, t]$, where $\mathrm{ACB}$ is the function computed by Algorithm 2.

Then,

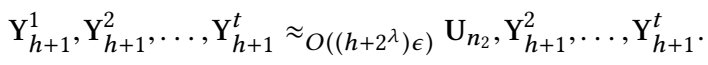




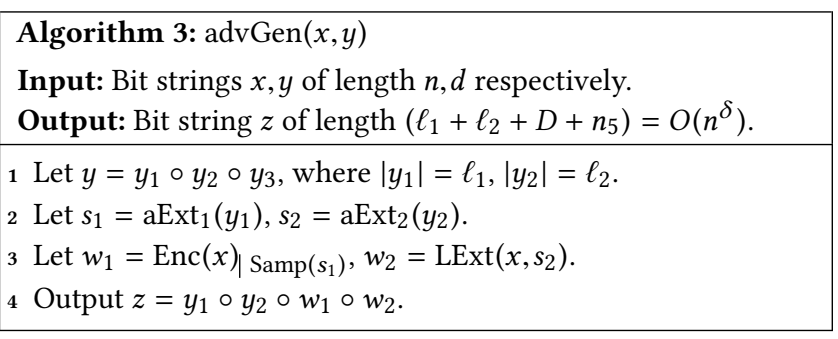

\subsection{Seedless Advice Generators for Affine Sources against Affine Adversaries}

Chattopadhyay, Goyal and Li [7] implicitly introduced objects called 'advice generators' in the context of constructing seeded non-malleable extractors. Cohen [17] formally defined these object, and follow-up works on non-malleable extractors also used explicit constructions of advice generators [8, 16]. Informally, an advice generator takes as input a weak source $\mathrm{X}$ and an independent seed $\mathrm{Y}$ to produce a short string such that for any random variable $\mathrm{Y}^{\prime}$, such that $\mathrm{Y}^{\prime} \neq \mathrm{Y}$, we have $\operatorname{advGen}(\mathrm{X}, \mathrm{Y}) \neq \operatorname{advGen}\left(\mathrm{X}, \mathrm{Y}^{\prime}\right)$ (with high probability).

Here we construct an advice generator for an affine source $\mathbf{X}$ assuming access to a short uniform seed $\mathrm{Y}=L(\mathrm{X})$, where $L$ is a linear function. Further we assume that $\mathrm{X}$ is tampered by an affine adversary $\mathcal{A}$ such that $\mathcal{A}$ has no fixed points. Note that unlike in previous work, the seed $\mathrm{Y}$ here is not independent of $\mathrm{X}$ and is in fact a deterministic function of $\mathrm{X}$.

THEOREM 5.3. There exists a constant $C$ such that for all $n>0$, any constant $\delta>0$ and $d \geq C n^{\delta}$, there exists an efficiently computable function advGen : $\{0,1\}^{n} \times\{0,1\}^{d} \rightarrow\{0,1\}^{\ell}, \ell=O\left(n^{\delta}\right)$ such that if $\mathrm{X}$ is an affine source on $\{0,1\}^{n}$ with entropy at least $n^{10 \delta}$ and $\mathrm{Y}=\mathcal{A}(\mathrm{X})$ is uniform on d bits, where $A$ is some affine function, and $\mathrm{X}^{\prime}=L(\mathrm{X}), \mathrm{Y}^{\prime}=\mathcal{A}\left(\mathrm{X}^{\prime}\right)$ for some affine function $L:\{0,1\}^{n} \rightarrow\{0,1\}^{n}$ with no fixed points, then $\operatorname{Pr}\left[\operatorname{advGen}(\mathrm{X}, \mathrm{Y}) \neq \operatorname{advGen}\left(\mathrm{X}^{\prime}, \mathrm{Y}^{\prime}\right)\right] \geq$ $1-2^{-n^{\Omega(1)}}$.

Proof. For easier presentation, we use the following notation: For any r.v $\mathbf{W}=f(\mathbf{X})$, we use $\mathbf{W}^{\prime}$ to denote $f\left(\mathbf{X}^{\prime}\right)$.

We setup some ingredients for our construction.

- Let $n_{1}=n^{\delta}, n_{2}=n-n^{\delta}$.

- Let Enc : $\{0,1\}^{n} \rightarrow\{0,1\}^{n_{3}}$ be the encoding function of an asymptotically good linear binary code with constant relative rate $1 / \lambda$ and constant relative distance $\beta$ (e.g, see [4]). Thus $n_{3}=\lambda n$.

- Let $n_{3}=2^{n_{4}}$. Let $\operatorname{Ext}_{1}:\{0,1\}^{n_{1}} \times\{0,1\}^{d_{1}} \rightarrow\{0,1\}^{n_{4}}$ be the seeded extractor from Theorem 4.4 set to extract from minentropy $n_{1} / 2$ with error $\epsilon=\beta / 2$. Thus $d_{1}=\log \left(n_{1}\right)+O(1)$.

- Let Samp : $\{0,1\}^{n_{1}} \rightarrow\left(\{0,1\}^{n_{4}}\right)^{D}$, be the sampler from Theorem 4.7 using $\operatorname{Ext}_{1}$. Thus $D=2^{d_{1}}=C_{1} n_{1}$ for some constant $C_{1}$.

- Let $\ell_{1}=C_{1} n_{1}$ for a large enough constant $C_{1}$ such that aExt $_{1}:\{0,1\}^{\ell_{1}} \rightarrow\{0,1\}^{n_{1}}$ is an affine extractor from Theorem 4.5 set to extract from min-entropy $\ell_{1} / 2$ with error $2^{-\Omega\left(\ell_{1}\right)}$

- Let LExt : $\{0,1\}^{n} \times\{0,1\}^{d_{2}} \rightarrow\{0,1\}^{n_{5}}, n_{5}=n^{\delta / 2}$, be a linear seeded extractor from Theorem 4.2 set to extract from min-entropy $\ell_{1} / 4$ with error $2^{-n^{\delta / 10}}$. It follows that $d_{2}<n^{\delta / 4}$.

- Let $\ell_{2}=C_{2} d_{2}$ for a large enough constant $C_{2}$ such that aExt $_{2}:\{0,1\}^{\ell_{2}} \rightarrow\{0,1\}^{d_{2}}$ is an affine extractor from Theorem 4.5 set to extract from min-entropy $\ell_{2} / 2$ with error $2^{-\Omega\left(\ell_{2}\right)}$

We prove that $\operatorname{Pr}\left[\operatorname{advGen}(\mathrm{X}, \mathrm{Y}) \neq \operatorname{advGen}\left(\mathrm{X}^{\prime}, \mathrm{Y}^{\prime}\right)\right]>1-2^{-n^{\Omega(1)}}$, where advGen is the function computed by Algorithm 3.

Assume that $\mathrm{Y}_{1}=\mathrm{Y}_{1}^{\prime}$ and $\mathrm{Y}_{2}=\mathrm{Y}_{2}^{\prime}$, since otherwise we directly have $\mathbf{Z} \neq \mathbf{Z}^{\prime}$. Without loss of generality assume $\mathrm{X}$ is uniform on some subspace of dimension $k$. Let $\ell=\ell_{1}+\ell_{2}$. Further let $\overline{\mathrm{Y}_{1}}=\mathrm{Y}_{1} \circ 0^{n-\ell_{1}}$ and $\overline{\mathrm{Y}_{2}}=0^{\ell_{1}} \circ \mathrm{Y}_{2} \circ 0^{n-\ell}$. Using simple linear algebra, it follows that there exist disjoint subspaces $A$ and $B$ such that $\mathbf{X}=\mathbf{A}+\mathbf{B}, \operatorname{dim}(\mathbf{A})=\ell, \operatorname{dim}(\mathbf{B})=k-\ell$ and $T\left(\overline{\mathrm{Y}_{1}}+\overline{\mathrm{Y}_{2}}\right)=\mathbf{A}$ for some linear function $T:\{0,1\}^{n} \rightarrow\{0,1\}^{n}$.

We have,

$$
\begin{aligned}
\mathbf{W}_{1}-\mathbf{W}_{1}^{\prime} & =\operatorname{Enc}(\mathbf{X})_{\mid \operatorname{Samp}\left(\mathrm{S}_{1}\right)}-\operatorname{Enc}\left(\mathbf{X}^{\prime}\right)_{\mid \operatorname{Samp}\left(\mathrm{S}_{1}\right)} \\
& =\operatorname{Enc}\left(\mathbf{X}-\mathbf{X}^{\prime}\right)_{\mid \operatorname{Samp}\left(\mathrm{S}_{1}\right)},
\end{aligned}
$$

and

$$
\begin{aligned}
\mathbf{W}_{2}-\mathbf{W}_{2}^{\prime} & =\operatorname{LExt}\left(\mathbf{X}, \mathbf{S}_{2}\right)-\operatorname{LExt}\left(\mathbf{X}^{\prime}, \mathbf{S}_{2}\right) \\
& =\operatorname{LExt}\left(\mathbf{X}-\mathbf{X}^{\prime}, \mathbf{S}_{2}\right)
\end{aligned}
$$

Further,

$$
\begin{aligned}
\mathbf{X}-\mathbf{X}^{\prime} & =\mathbf{A}+\mathbf{B}-L(\mathbf{A})-L(\mathbf{B}) \\
& =T\left(\overline{\mathbf{Y}_{1}}\right)-L\left(T\left(\overline{\mathrm{Y}_{1}}\right)\right)+T\left(\overline{\mathrm{Y}_{2}}\right)-L\left(T\left(\overline{\mathrm{Y}_{2}}\right)\right)+\mathbf{B}-L(\mathbf{B})
\end{aligned}
$$

Now consider the following cases.

- $H_{\infty}\left(T\left(\overline{\mathrm{Y}_{1}}\right)-L\left(T\left(\overline{\mathrm{Y}_{1}}\right)\right)\right) \leq \ell_{1} / 2$.

In this case, we can fix $T\left(\overline{\mathrm{Y}_{1}}\right)-L\left(T\left(\overline{\mathrm{Y}_{1}}\right)\right)$, and it follows that $\mathrm{Y}_{1}$ has min-entropy at least $\ell_{1} / 2$ after this fixing. Further note that $Y_{1}$ is still an affine source, and hence it follows that $\mathrm{S}_{1}$ is $2^{-\Omega\left(\ell_{1}\right)}$-close to uniform. Further fix $\mathbf{B}, \mathbf{Y}_{2}$, noting that it is independent of $\mathrm{Y}_{1}$. This in fact fixes $X-X^{\prime}$. Since $X \neq X^{\prime}$, it follows that $\operatorname{Enc}(X)$ differs from $\operatorname{Enc}\left(\mathbf{X}^{\prime}\right)$ in at least $\beta$ fraction of the coordinates. Using Theorem 4.7, it follows that with probability at least $1-2^{-\Omega\left(n_{1}\right)}$, Samp $\left(\mathrm{S}_{1}\right)$ intersects one of the coordinates on which $\operatorname{Enc}(\mathrm{X})$ differs from $\operatorname{Enc}\left(\mathrm{X}^{\prime}\right)$ (and thus $\mathbf{W}_{1} \neq \mathbf{W}_{1}^{\prime}$ ).

- $H_{\infty}\left(T\left(\overline{\mathrm{Y}_{1}}\right)-L\left(T\left(\overline{\mathrm{Y}_{1}}\right)\right)\right)>\ell_{1} / 2$.

We have,

$$
\begin{aligned}
\mathbf{W}_{2}-\mathbf{W}_{2}^{\prime} & =\operatorname{LExt}\left(\mathbf{X}-\mathbf{X}^{\prime}, \mathrm{S}_{2}\right) \\
& =\operatorname{LExt}\left(T\left(\overline{\mathrm{Y}_{1}}\right)-L\left(T\left(\overline{\mathrm{Y}_{1}}\right)\right), \mathrm{S}_{2}\right)+ \\
& \operatorname{LExt}\left(T\left(\overline{\mathrm{Y}_{2}}\right)-L\left(T\left(\overline{\mathrm{Y}_{2}}\right)\right), \mathrm{S}_{2}\right)+\operatorname{LExt}\left(\mathbf{B}-L(\mathbf{B}), \mathrm{S}_{2}\right)
\end{aligned}
$$

It follows that $\operatorname{LExt}\left(T\left(\overline{\mathrm{Y}_{1}}\right)-L\left(T\left(\overline{\mathrm{Y}_{1}}\right)\right), \mathrm{S}_{2}\right)$ is $2^{-n^{\Omega(1)}}$-close to uniform. We fix $\mathrm{S}_{2}$ since LExt is a strong seeded extractor, and thus $\operatorname{LExt}\left(T\left(\overline{\mathrm{Y}_{1}}\right)-L\left(T\left(\overline{\mathrm{Y}_{1}}\right)\right), s_{2}\right)$ is now a deterministic function of $Y_{1}$. We now fix $Y_{2}$, B using the fact that they are independent of $\mathrm{Y}_{1}$. Thus, after these fixings $\mathbf{W}_{2}-\mathbf{W}_{2}^{\prime}=$ $\operatorname{LExt}\left(T\left(\overline{\mathrm{Y}_{1}}\right)-L\left(T\left(\overline{\mathrm{Y}_{1}}\right)\right), s_{2}\right)+\alpha$ (for some constant $\alpha \in$ $\left.\{0,1\}^{n_{5}}\right)$, and hence is $2^{-n^{\Omega(1)}}$ close to uniform on average. Thus, $\operatorname{Pr}\left[\mathbf{W}_{2}=\mathbf{W}_{2}^{\prime}\right] \leq 2^{-n^{\Omega(1)}}+2^{-n_{5}}$. 


\subsection{The Extractor Construction}

Theorem 5.4. There exists a constant $C_{1}$ such that for all $n, k$, $w>0$ and any $\delta>0$ with $w<n^{\beta}, \beta=\delta /\left(3 C_{1}\right)$ and $k \geq n^{C_{1} \delta}$, there exists an efficient function anmExt : $\{0,1\}^{n} \rightarrow\{0,1\}^{m}, m=k^{\Omega(1)}$, such that if $\mathrm{X}$ is a $w$-affine source with min-entropy at least $k$ and $\mathcal{A}:\{0,1\}^{n} \rightarrow\{0,1\}^{n}$ is an affine function with no fixed points, then $\left|\operatorname{anmExt}(\mathrm{X}), \operatorname{anmExt}(\mathcal{A}(\mathrm{X}))-\mathrm{U}_{m}, \operatorname{anmExt}(\mathcal{A}(\mathrm{X}))\right| \leq 2^{-n^{\Omega(1)}}$

We use the rest of the section to prove the above theorem. We reuse the following notation from previous section: For any r.v $\mathbf{W}=f(\mathbf{X})$, we use $\mathbf{W}^{\prime}$ to denote $f\left(\mathbf{X}^{\prime}\right)$. We first set up the required ingredients with appropriate parameters.

- Let LCon : $\{0,1\}^{n} \rightarrow\{0,1\}^{n_{1}}$ be a linear condenser from Theorem 5.1 set to work for min-entropy $k_{1}=n^{\delta}$ and parameter $\alpha$ (which we fix below). Thus $n_{1}=w k_{1}^{\alpha} \log n$.

- Let LExt $2:\{0,1\}^{n} \times\{0,1\}^{d_{2}} \rightarrow\{0,1\}^{n_{2}}, n_{2}=k^{1-2 \delta}$, be a linear seeded extractor from Theorem 4.2 set to extract from min-entropy $k_{3}=k / 2$ with error $\epsilon_{2}=2^{-n^{\delta_{1}}}, \delta_{1}=$ $\alpha \delta / 10$. Thus $d_{2}=n^{2 \delta_{1}} \log ^{2} n<k_{1}^{\alpha / 2}$.

- Let $\operatorname{LExt}_{1}:\{0,1\}^{n_{1}} \times\{0,1\}^{d_{1}} \rightarrow\{0,1\}^{d_{2}}$ be a linear seeded extractor from Theorem 4.2 set to extract from min-entropy $k_{2}=k_{1}^{\alpha}$ with error $\epsilon_{1}=1 / 20$. It follows that $d_{1}=O\left(\log \left(n_{1}\right)\right)$. Let $D_{1}=2^{d_{1}}$. Thus $D_{1}=n_{1}^{C_{1}}$, for some constant $C_{1}$.

- Fix $\alpha=1 /\left(2 C_{1}\right), \beta=\delta /\left(2 C_{1}\right)$.

- Let advGen : $\{0,1\}^{n} \times\{0,1\}^{d_{3}} \rightarrow\{0,1\}^{a}$ be the advice generator from Theorem 5.3 set to work with parameter $\delta_{5.3}=\delta$. Thus we can fix $d_{3}=C_{5.3} n^{\delta_{5.3}}$.

- Let ACB : $\{0,1\}^{n_{2}} \times\{0,1\}^{n} \times\{0,1\}^{h} \rightarrow\{0,1\}^{m}, h=a+d_{1}$, be the correlation breaker from Theorem 5.2 setup using the following components:

- $\operatorname{LExt}_{1, \mathrm{ACB}}:\{0,1\}^{n} \times\{0,1\}^{d_{4}} \rightarrow\{0,1\}^{d_{4}}$ and LExt $2, \mathrm{ACB}:$ $\{0,1\}^{m} \times\{0,1\}^{d_{4}} \rightarrow\{0,1\}^{d_{4}}$ be $\left(k_{\mathrm{ACB}}=C_{2} a^{2} D_{1}^{2} \log ^{2} n\right.$, $\left.\epsilon_{\mathrm{ACB}}=2^{-4 a D_{1}}\right)$-strong linear seeded extractors, $m=$ $k_{\mathrm{ACB}}+C_{2} D_{1} d_{4}+4 a D_{1}$ for some large enough constant $C_{2}$, instantiated from Theorem 4.2. Thus, $d_{4}<k_{\mathrm{ACB}} / 2$, for an appropriately chosen $C_{2}$.

- $\operatorname{LExt}_{3, \mathrm{ACB}}:\{0,1\}^{n_{1}} \times\{0,1\}^{d_{4}} \rightarrow\{0,1\}^{m}$ be a $\left(k_{2, \mathrm{ACB}}=\right.$ $\left.m^{1.1}, \epsilon_{\mathrm{ACB}}\right)$-strong linear seeded extractor.

The following bounds follow directly from our choice of parameters.

(1) $D_{1} \leq n^{\delta}, a \leq n^{\delta}, D_{1} n_{2} \leq k^{1-\delta}$,

(2) $k_{\mathrm{ACB}}<m<n^{5 \delta}$.

\begin{tabular}{|l|}
\hline Algorithm 4: $\operatorname{anmExt}(x)$ \\
Input: Bit string $x$ of length $n$. \\
Output: Bit string $z$ of length $m$. \\
\hline 1 Let $v=\operatorname{LCon}(x)$. \\
2 Let $r$ be a $D_{1} \times n_{2}$ matrix, whose $i$ 'th row $r_{i}$, is \\
LExt $2\left(x, \operatorname{LExt}_{1}(v, i)\right)$. \\
3 Let $s_{i}$ be a $D_{1} \times m$ matrix whose $i$ 'th row $s_{i}$, is \\
ACB $\left(r_{i}, x, \operatorname{advGen}\left(x\right.\right.$, Slice $\left.\left.\left(r_{i}, d_{3}\right)\right) \circ i\right)$. \\
4 Output $z=\oplus_{j=1}^{D_{1}} s_{i}$. \\
\hline
\end{tabular}

Claim 5.5. Conditioned on $\mathrm{V}, \mathrm{V}^{\prime}$, the r.v $\mathrm{R}$ is $2^{-n^{\Omega(1)}}$-close to an affine somewhere random source.

Proof. Using Theorem 5.1 it follows that $\mathrm{V}$ is a $\left(n_{1}, k_{1}^{\alpha}\right)$-source. Now by Lemma 4.1, there exists a set $I \subseteq\left[D_{1}\right],|I| \geq 0.9 D_{1}$ such that for any $i \in[I], \operatorname{LExt}_{1}(\mathbf{V}, i)=\mathbf{U}_{d_{2}}$. We note that $\mathbf{V}, \mathbf{V}^{\prime}$ are obtained by applying linear functions on $\mathrm{X}$. Thus there exist disjoint subspaces $\mathbf{A}$ and $\mathbf{B}$ such that $\mathbf{X}=\mathbf{A}+\mathbf{B}, H_{\infty}(\mathbf{B}) \geq k-2 n_{1}$ and $\mathbf{B}$ is independent of $\mathbf{V}, \mathrm{V}^{\prime}$. Now for any $i \in I$, we have

$$
\begin{array}{r}
\mathbf{R}_{i}=\operatorname{LExt}_{2}\left(\mathbf{X}, \operatorname{LExt}_{1}(\mathbf{V}, i)\right)=\operatorname{LExt}_{2}\left(\mathbf{A}, \operatorname{LExt}_{1}(\mathbf{V}, i)\right)+ \\
\operatorname{LExt}_{2}\left(\mathbf{B}, \operatorname{LExt}_{1}(\mathbf{V}, i)\right) .
\end{array}
$$

Since B is independent of $\mathbf{V}$, it follows that $\operatorname{LExt}_{2}\left(\mathbf{B}, \operatorname{LExt}_{1}(\mathbf{V}, i)\right)$ is $\epsilon_{2}$-close to $\mathbf{U}_{n_{2}}$. Further, since LExt 2 is strong seeded, we fix $\operatorname{LExt}_{1}(\mathbf{V}, i)$. Thus, $\operatorname{LExt}_{2}\left(\mathbf{B}, \operatorname{LExt}_{1}(\mathbf{V}, i)\right)$ is now a deterministic function of $\mathbf{B}$. We also fix $\operatorname{LExt}_{2}\left(\mathbf{A}, \operatorname{LExt}_{1}(\mathbf{V}, i)\right)$ since it is independent of B. $\mathbf{R}_{i}$ remains $\epsilon_{2}$-close to $\mathbf{U}_{n_{2}}$ on average after these fixings. Further fix $\mathbf{V}, \mathbf{V}^{\prime}$ noting that it does not affect the distribution of $\mathbf{R}_{j}$. Each $\mathbf{R}_{i}$ is now a linear function of $\mathbf{X}$. Hence it follows that $\mathbf{R}$ is $\epsilon_{2}$-close to an affine somewhere random source.

Claim 5.6. $\left|\left(\mathbf{Z}, \mathbf{Z}^{\prime}\right)-\left(\mathbf{U}_{m}, \mathbf{Z}^{\prime}\right)\right|=2^{-n^{\Omega(1)}}$.

Proof. We fix $\mathbf{V}, \mathbf{V}^{\prime}$ and note by the previous claim that $\mathbf{R}$ is now an affine somewhere random source. Without loss of generality, suppose $\mathbf{R}_{1}$ is the random row. We claim that

$$
\begin{array}{r}
\mid \mathrm{S}_{1},\left\{\mathrm{~S}_{i}: i \in\left[2, D_{1}\right]\right\},\left\{\mathrm{S}_{i}^{\prime}: i \in\left[D_{1}\right]\right\}- \\
\mathbf{U}_{m},\left\{\mathrm{~S}_{i}: i \in\left[2, D_{1}\right]\right\},\left\{\mathrm{S}_{i}^{\prime}: i \in\left[D_{1}\right]\right\} \mid \leq 2^{-n^{\Omega(1)}} .
\end{array}
$$

Since Claim 5.6 is direct from this, we now focus on proving the above bound. Theorem 5.3 guarantees that $\operatorname{advGen}\left(\mathbf{X}\right.$, Slice $\left(\mathbf{R}_{1}\right.$, $\left.\left.d_{3}\right)\right) \neq \operatorname{advGen}\left(\mathbf{X}^{\prime}, \operatorname{Slice}\left(\mathbf{R}_{j}^{\prime}, d_{3}\right)\right)$ (with probability at least $\left.1-2^{-n^{\Omega(1)}}\right)$ for any $j \in\left[D_{1}\right]$. Fix the r.v's $\left\{\operatorname{advGen}\left(\mathbf{X}\right.\right.$, Slice $\left.\left.\left(\mathbf{R}_{i}, d_{3}\right)\right): i \in\left[D_{1}\right]\right\}$, $\left\{\operatorname{advGen}\left(\mathbf{X}^{\prime}, \operatorname{Slice}\left(\mathbf{R}_{i}^{\prime}, d_{3}\right)\right): i \in\left[D_{1}\right]\right\}$, and the following hold with probability at least $1-2^{-n^{\Omega(1)}}$ :

- $\mathbf{R}_{1}$ has min-entropy at least $n_{2}-2 a D_{1}$

- $\mathrm{X}$ remains an affine source and has min-entropy at least $k-2 a D_{1}-n_{1}$

- $\mathbf{R}$ and $\mathbf{R}^{\prime}$ are obtained by applying affine functions on $\mathbf{X}$

- $\operatorname{advGen}\left(\mathbf{X}, \operatorname{Slice}\left(\mathbf{R}_{1}, d_{3}\right)\right) \neq \operatorname{advGen}\left(\mathbf{X}, \operatorname{Slice}\left(\mathbf{R}_{j}^{\prime}, d_{3}\right)\right)$

Thus we can write $\mathbf{X}=\overline{\mathbf{A}}+\overline{\mathbf{B}}$ such that $\overline{\mathbf{B}}$ is independent of $\left\{\mathbf{R}, \mathbf{R}^{\prime}\right\}$ and $H_{\infty}(\overline{\mathbf{B}})=k_{b} \geq k-2 a D_{1}-n_{1}-2 D_{1} n_{2}>k / 2$. Let $\lambda=2 a D_{1}$. The claim is now direct from Theorem 5.2 noting that the following hold:

- $k_{b} \geq C\left(k_{\mathrm{ACB}}+d_{4} D_{1} h+\log \left(1 / \epsilon_{\mathrm{ACB}}\right)\right)$, for any constant $C$.

- $n_{2} \geq C\left(k_{\mathrm{ACB}}+d_{4} D_{1} h+h D_{1} m^{2}+\log \left(1 / \epsilon_{\mathrm{ACB}}\right)\right)$, for any constant $C$.

- $\left(2^{\lambda}+h\right) \epsilon=2^{-n^{\Omega(1)}}$. 


\section{SEEDLESS NON-MALLEABLE EXTRACTORS FOR LOCAL AND AC ${ }^{0}$ ADVERSARIES}

The main results in this section is are seedless non-malleable extractor against $\mathrm{t}$-local and $\mathrm{AC}^{0}$ functions. Our main idea is to use techniques developed by Viola [33], where he designed extractors for sources sampled by $\mathrm{AC}^{0}$ circuits. We first reduce the problem of constructing non-malleable extractors against $\mathrm{AC}^{0}$ adversaries to the problem of constructing non-malleable extractors against local adversaries. Next, we show reduce the problem of constructing non-malleable extractors for local adversaries to the problem of constructing extractors for low-weight affine sources against affine adversaries. Note that in Theorem 5.4 we exactly construct such non-malleable extractors, and hence this gives non-malleable extractors against local and $\mathrm{AC}^{0}$ functions.

The following are the main results of this section. We derive these results assuming the reductions in Section 6.1 and Section 6.2 (see Lemma 6.2 and Lemma 6.3).

TheOREM 6.1. For any $\delta>0$ and for all $n>0$, there exists an efficient function localnmExt : $\{0,1\}^{n} \rightarrow\{0,1\}^{m}, m=n^{\Omega(1)}$, such that if $\mathrm{X}$ is an oblivious bit-fixing source on $n$ bits with min-entropy $k$ and $f:\{0,1\}^{n} \rightarrow\{0,1\}^{n}$ is a $t$-local function, $t \leq k / n^{\frac{1}{2}+\delta}$, then there exists a distribution $\mathrm{Y}$ on $\{0,1\}^{m} \cup\left\{\right.$ same $\left.^{\star}\right\}$ that is independent of $\mathrm{X}$, and

$\left.\mid \operatorname{localnmExt}(\mathbf{X}), \operatorname{loc} \operatorname{lnm} \operatorname{Ext}(f(\mathbf{X}))-\mathbf{U}_{m}, \operatorname{copy}\left(\mathrm{Y}, \mathbf{U}_{m}\right)\right) \mid \leq 2^{-n^{\Omega(1)}}$.

Proof. Let anmExt : $\{0,1\}^{n} \rightarrow\{0,1\}^{m}, m=n^{\gamma}$ for some small enough constant $\gamma$, be the non-malleable extractor from Theorem 5.4 set to extract from min-entropy $n^{\delta}-n^{\delta / 2}$ with the parameter $w$ set to $n^{\delta / 2}$ and error $2^{-n^{\Omega(1)}}$. Define localnmExt $:=$ anmExt. Using Lemma 6.3, it follows that $(\mathrm{X}, f(\mathrm{X}))$ is a convex combination of sources of the form $\left(\mathrm{Z}_{i}, A_{i}\left(\mathrm{Z}_{i}\right)\right)$, such that each $\mathrm{Z}_{i}$ is an oblivious bit-fixing source with min-entropy at least $n^{2 \delta} / 2$ and $A_{i}$ is a 1-local function. Now by Lemma 3.1, it follows that anmExt is a non-malleable extractor for $\mathbf{Z}_{i}$ against 1-local tampering with error $2^{-n^{\Omega(1)}}+n 2^{-n^{\delta / 2}}$. The theorem now follows by a convex combination argument.

Theorem 6.2. For all $n>0$ and any $d=O(1)$, there exists an efficient function acnmExt : $\{0,1\}^{n} \rightarrow\{0,1\}^{m}, m=n^{\Omega(1)}$, such that if $\mathrm{X}$ is uniform on $n$ bits and $C:\{0,1\}^{n} \rightarrow\{0,1\}^{n}$ is an $\mathrm{AC}^{0}$ circuit, there exists a distribution $\mathrm{Y}$ on $\{0,1\}^{m} \cup\left\{\right.$ same $\left.^{\star}\right\}$ that is independent of $\mathrm{X}$, and

$$
\left.\mid \operatorname{acnmExt}(\mathrm{X}), \operatorname{acnmExt}(C(\mathrm{X}))-\mathbf{U}_{m}, \operatorname{copy}\left(\mathrm{Y}, \mathbf{U}_{m}\right)\right) \mid \leq \frac{1}{n^{\Omega(\log n)}} .
$$

Proof. Let localnmExt be the extractor from Theorem 6.1 set to extract from min-entropy $k=n^{1-\gamma}$, for a small enough constant $\gamma>$ 0 and locality parameter $t$ set to $n^{\gamma}$. Define acnmExt $=$ localnmExt. Now by Lemma 6.2 , it follows that $(\mathrm{X}, C(\mathrm{X}))$ is $n^{-\Omega(\log n)}$-close to a convex combination of distributions of the form $\left(\mathbf{Z}_{i}, f_{i}\left(\mathbf{Z}_{i}\right)\right)$, where each $Z_{i}$ is an oblivious bit-fixing source with min-entropy at least $n^{1-\gamma}$ and $f_{i}$ is a $n^{\gamma}$-local function. The theorem now follows directly.

\subsection{A Reduction from $A C^{0}$ to Local Adversaries}

The reduction from $\mathrm{AC}^{0}$ to local adversaries is based on the well known switching lemma [25]. We first recall the definition of a random restriction. A $p$-restriction $\rho$ acting on a string $x \in\{0,1\}^{n}$ independently fixes each bit to 0 with probability $(1-p) / 2$, to 1 with probability $(1-p) / 2$ and leaves it unfixed with probability $p$. Let $P_{p}$ denote the set of all $p$-restrictions acting on $n$ bits. For a function $f: z o^{n} \rightarrow\{0,1\}^{m}$, and a $p$-restriction $\rho$, let $f_{\rho}$ denote the function after applying the restriction $\rho$.

LEMMA 6.1 ([25]). Let $f:\{0,1\}^{n} \rightarrow\{0,1\}$ be a function computed by a size $s \mathrm{AC}^{0}$ circuit of depth $d$. Then, for a uniformly drawn $p$ restriction $\rho$ from $P_{p}$, we have

$$
\underset{\rho}{\operatorname{Pr}}\left[f\left(x_{\mid \rho}\right) \text { is not a } 2^{t} \text {-local function }\right] \leq s\left(9 p^{1 / d} t\right)^{t} .
$$

LEMma 6.2. For any $d=O(1)$ and any constants $\delta, \gamma>0$ the following holds: Let $C:\{0,1\}^{n} \rightarrow\{0,1\}^{n}$ be any $\mathrm{AC}^{0}$ circuit of size $n^{d}$ and $\mathrm{X}$ be the uniform distribution on $n$ bits. Then there exists oblivious bit-fixing sources $\mathbf{X}_{1}, \ldots, \mathbf{X}_{\ell}$ on $\{0,1\}^{n}$, and $f_{1}:\{0,1\}^{n} \rightarrow\{0,1\}^{n}$, $\ldots, f_{\ell}:\{0,1\}^{n} \rightarrow\{0,1\}^{n}$, such that

- $H_{\infty}\left(\mathbf{X}_{i}\right) \geq n^{1-\gamma}$ for each $i \in[\ell]$,

- Each $f_{i}$ is a $n^{\delta}$-local function.

- $(\mathrm{X}, C(\mathrm{X}))$ is $1 / n^{\Omega(\log n)}$-close to a convex combination of the distributions $\left(\mathbf{X}_{i}, f_{i}\left(\mathbf{X}_{i}\right)\right)$.

\subsection{A Reduction from Local to Affine Adversaries}

Our reduction from local to affine adversaries uses some ideas from [33] but the analysis is much simpler.

LEMma 6.3. Let $\mathrm{X}$ be an oblivious bit-fixing source on $n$ bits with min-entropy $k$, and let $f:\{0,1\}^{n} \rightarrow\{0,1\}^{n}$ be any $t$-local function. Then there exists sources $\mathbf{Z}^{1}, \ldots, \mathbf{Z}^{\ell}$, each on $n$ bits, and affine functions $A_{1}, \ldots, A_{\ell}$, such that

- $(\mathbf{X}, f(\mathbf{X}))$ is a convex combination of $\left(\mathbf{Z}^{i}, A_{i}\left(\mathbf{Z}^{i}\right)\right), i \in[\ell]$.

- Each $\mathrm{Z}_{i}$ is an oblivious bit-fixing source with min-entropy at least $k^{2} /\left(8 t^{2} n\right)$.

Proof SкeTch. Without loss of generality, suppose $x_{1}, \ldots, x_{k}$ are the random bits in the oblivious source $X$. Let $\mathrm{Y}=f(\mathrm{X})$. Consider a bipartite graph $G$ with left nodes $\mathcal{L}=\left\{x_{1}, \ldots, x_{k}\right\}$ and right nodes $\mathbb{R}=\left\{y_{1}, \ldots, y_{n}\right\}$, with an edge present between $x_{i}$ and $y_{j}$ if the bit $y_{j}$ depends on $x_{i}$.

By a markov argument, it follows that at most $k / 2$ of the left vertices have degree more than $d_{\ell}$. Let $\mathcal{L}^{\prime}$ set of vertices on the left with degree more $d_{\ell}$. Let $k^{\prime}=k^{2} /\left(8 t^{2} n\right)$. For any set $S \subset[n]$, we use $x_{S}$ to denote the set $\left\{x_{i}: i \in S\right\}$. We use the following iterative process:

(1) Uniformly sample, and fix the variables in $\mathcal{L}^{\prime}$.

(2) Let $\mathcal{L}_{0}=\mathcal{L} \backslash \mathcal{L}^{\prime}, V_{0}=\emptyset$.

(3) For $i=1$ to $k^{\prime}$, do:

(4) Pick some $x_{j}$ in $\mathcal{L}_{i-1}$, and uniformly sample and fix the variables in $N(N(x))$.

(5) Set $\mathcal{L}_{i}$ to $\mathcal{L}_{i-1} \backslash N\left(N\left(x_{j}\right)\right)$, and set $V_{i}=V_{i-1} \cup\{j\}$.

(6) Set the bits $k+1, \ldots, x_{n}$ consistent with $\mathrm{X}$.

(7) Sample uniformly and fix the unfixed variables in $\left\{x_{j}: j \in\right.$ $\left.[n] \backslash V_{k^{\prime}}\right\}$.

(8) Let $V_{k^{\prime}}^{\prime}=[n] \backslash V_{k^{\prime}}$. 
(9) Let $A:\{0,1\}^{n} \rightarrow\{0,1\}^{n}$ be the function defined in the following way: For any $w \in\{0,1\}^{n}, A\left(w_{1}, \ldots, w_{n}\right)=f\left(x_{V_{k^{\prime}}^{\prime}} \circ\right.$ $\left.w_{V_{k^{\prime}}}\right)$.

(10) Sample $\left\{x_{j}: j \in V_{k^{\prime}}\right\}$ uniformly.

(11) Output $(x, A(x))$.

We refer the reader to the full version for a proof of correctness of the above procedure.

\section{SEEDLESS NON-MALLEABLE EXTRACTORS FOR AFFINE SOURCES AGAINST AFFINE ADVERSARIES}

In this section we construct seedless non-malleable extractors for arbitrary affine sources against affine adversaries. We construct extractors for affine sources on $n$ bits with min-entropy at least $n-n^{\delta}$ and error $2^{-n^{\Omega(1)}}$ assuming that the affine adversary has no fixed points. By Lemma 3.2, it directly implies affine extractors for affine sources with min-entropy at least $n-n^{\delta} / 2$ and error $2^{-n^{\Omega(1)}}+n 2^{-n^{\delta} / 2}$ against arbitrary affine functions. The following is the main theorem in this section.

Theorem 7.1. For all $n, k>0$, any $\delta>0$ and $k \geq n-n^{\delta}$, there exists an efficient function anmExt : $\{0,1\}^{n} \rightarrow\{0,1\}^{m}$ such that if $\mathrm{X}$ is an affine source with min-entropy at least $k$ and $\mathcal{A}:\{0,1\}^{n} \rightarrow$ $\{0,1\}^{n}$ is an affine function with no fixed points, then

$$
\left|\operatorname{anmExt}(\mathbf{X}), \operatorname{anmExt}(\mathcal{A}(\mathbf{X}))-\mathbf{U}_{m}, \operatorname{anmExt}(\mathcal{A}(\mathbf{X}))\right| \leq 2^{-n^{\Omega(1)}} .
$$

The construction is almost the same as the extractor construction in Section 5.4, and we reuse components and parameters that were setup in Section 5.4. We omit the proof of Theorem 7.1 since it follows along the lines of the proof of Theorem 5.4.

\begin{tabular}{|l|}
\hline Algorithm 5: anmExt $(x)$ \\
Input: Bit string $x$ of length $n$. \\
Output: Bit string $z$ of length $m$. \\
\hline 1 Let $v=\operatorname{Slice}\left(x, n_{1}\right)$. \\
2 Let $r$ be a $D_{1} \times n_{2}$ matrix, whose $i$ 'th row $r_{i}$, is \\
LExt $2\left(x, \operatorname{Lxt}_{1}(v, i)\right)$. \\
3 Let $s_{i}$ be a $D_{1} \times m$ matrix whose $i$ 'th row $s_{i}$, is \\
ACB $\left(r_{i}, x, \operatorname{advGen}\left(x, \operatorname{Slice}\left(r_{i}, d_{3}\right)\right) \circ i\right)$. \\
4 Output $z=\oplus_{j=1}^{D_{1}} s_{i}$. \\
\hline
\end{tabular}

\section{EFFICIENT SAMPLING ALGORITHMS}

In this section we suitably modify the non-malleable extractor anmExt : $\{0,1\}^{n} \rightarrow\{0,1\}^{m}$ from Algorithm 4 and the advice generator advGen from Algorithm 3, and present an efficient algorithm that on any input $z \in\{0,1\}^{m}$ samples from a distribution that is uniform on the set anmExt ${ }^{-1}(z)$. Since our other non-malleable extractors constructions are either reductions to anmExt or are very similar to the construction of anmExt, we do not explicitly present sampling algorithms for these constructions.

\subsection{The Modified Extractor}

We now describe the construction of the extractor ianmExt : $\{0,1\}^{n}$ $\rightarrow\{0,1\}^{m}$ which takes as input $x \in\{0,1\}^{n}$ and outputs $z \in\{0,1\}^{m}$. We refer the reader to the full version for a proof of correctness.

We use the following notation: for any linear map $L:\{0,1\}^{a} \rightarrow$ $\{0,1\}^{b}$, given by $L(\alpha)=M \alpha$ for some $b \times a$ matrix $M$, let $\operatorname{con}_{L}$ be a maximal set of linearly independent rows of $M$.

(1) Let LCon : $\{0,1\}^{n} \rightarrow\{0,1\}^{n_{1}}$ be a linear condenser from Theorem 5.1 set to work for min-entropy $k_{1}=n^{\delta}$ and parameter $\alpha$ (which we fix below). Thus $n_{1}=w k_{1}^{\alpha} \log n$. Let

$$
v=\operatorname{LCon}(x) \text {. }
$$

(2) Let $d_{2}$ be a parameter which we set below. Let LExt 1 : $\{0,1\}^{n_{1}} \times\{0,1\}^{d_{1}} \rightarrow\{0,1\}^{d_{2}}$ be a linear seeded extractor from Theorem 4.2 set to extract from min-entropy $k_{2}=$ $k_{1}^{\alpha} / 2$ with error $\epsilon_{1}=1 / 20$. Thus $d_{1}=O\left(\log n_{1}\right)$. Let $D_{1}=$ $2^{d_{1}}$. Thus, $D_{1}=n_{1}^{C_{1}}$ for some constant $C_{1}$. Let $r^{\prime}$ be the $D_{1} \times n_{1}$ matrix whose $i$ 'th row $r_{i}^{\prime}$ is defined as

$$
r_{i}^{\prime}=\operatorname{LExt}_{1}(v, i) \text {. }
$$

(3) Let LExt $2:\{0,1\}^{n} \times\{0,1\}^{d_{2}} \rightarrow\{0,1\}^{n_{2}}, n_{2}=k^{1-2 \delta}$, be a linear seeded extractor from Theorem 4.2 set to extract from min-entropy $k_{3}=k / 2$, and error $\epsilon_{2}=2^{-n^{\delta_{1}}}, \delta_{1}=$ $\alpha \delta / 10$. Thus $d_{2}=n^{2 \delta_{1}} \log ^{2} n<\sqrt{k_{2}}$.

(4) Set $\alpha=1 /\left(10 C_{1}\right)$.

(5) Let $n_{3}=n_{2} /\left(2 D_{1}\right)$ and $D^{\prime}=0.9 D_{1}$. We now define a sequence of extractors $\left\{\mathrm{LExt}_{2, i}^{v}\right\}_{i=1}^{D^{\prime}}$ in the following iterative way:

(a) Let $i \leftarrow 1, \mathcal{L} \leftarrow$ con $_{\mathrm{LCon}}$, counter $\leftarrow 0$, rowlist $\leftarrow \emptyset$.

(b) while $i \leq D_{1}$ and counter $<D^{\prime}$ :

(i) Define the function $f_{i}(x)=\operatorname{LExt}_{2}\left(x, \operatorname{LExt}_{1}(v\right.$, $i)$ ). Note that $f_{i}:\{0,1\}^{n} \rightarrow\{0,1\}^{n_{2}}$ is a linear function. If $f_{i}\left(\{0,1\}^{n}\right) \neq\{0,1\}^{n_{2}}$, go to (v).

(ii) Update counter $\leftarrow$ counter +1 , rowlist $\leftarrow$ rowlist $\cup\{i\}$.

(iii) For any $S \subseteq\left[n_{2}\right]$, let $f_{i, S}:\{0,1\}^{n} \rightarrow\{0,1\}^{|S|}$ be the projection of $f_{i}$ to the coordinates in $S$. If $S=\{j\}$ is a singleton, we use the notation $f_{i, j}$ instead of $f_{i,\{j\}}$. Thus, $f_{i, j}(x)=\left\langle q_{i, j}, x\right\rangle$ for some $q_{i, j} \in\{0,1\}^{n}$. Pick a subset $S_{i}^{v}$ of $\left[n_{2}\right]$, $\left|S_{i}^{v}\right|=n_{3}$ such that $\mathcal{L} \cup\left\{q_{i, j}: j \in S_{i}^{v}\right\}$ is a set of linearly independent vectors. Define

$\operatorname{LExt}_{2, i}^{v}(x, y)=\operatorname{LExt}_{2}(x, y)_{\mid S_{i}^{v}}$.

(iv) Update $\mathcal{L} \leftarrow \mathcal{L} \cup\left\{q_{i, j}: j \in S_{i}^{v}\right\}$.

(v) $i \leftarrow 1+1$

(6) If $\mid$ rowlist $\mid<D^{\prime}$, output

$$
z=0^{m} \text {. }
$$

(7) Let $r$ be the $D^{\prime} \times n_{3}$ matrix whose $i^{\prime}$ th row $r_{i}$ is defined as

$$
r_{i}=\operatorname{LExt}_{2, i}^{v}\left(x, \operatorname{LExt}_{1}(v, i)\right) .
$$

(8) Let $d_{3}=n^{\delta}$. For each $i \in\left[D^{\prime}\right]$, let $y_{1, i}=\operatorname{Slice}\left(r_{i}, 1, d_{3}\right)$.

(9) Let $d_{4}=d_{3}+d_{3}\left(D^{\prime}\right)^{2}$. Let $y_{2}=\operatorname{Slice}\left(r_{1}, d_{3}+1, d_{4}\right) \circ \ldots \circ$ Slice $\left(r_{D^{\prime}}, d_{3}+1, d_{4}\right)$. Let $n_{4}=\left(d_{4}-d_{3}\right) D$. 
(10) Let $C_{\mathrm{BCH}}$ be a BCH code with parameters: $\left[n_{\mathrm{BCH}}, n_{\mathrm{BCH}}-\right.$ $\left.t_{\mathrm{BCH}}\left(\log n_{\mathrm{BCH}}\right), 2 t_{\mathrm{BCH}}\right]_{2}, t_{\mathrm{BCH}}=\beta_{\mathrm{BCH}} \sqrt{n_{\mathrm{BCH}}} / 2$, for some small enough constant $\beta_{b c h}$, for some parameter $n_{\mathrm{BCH}}$. Let $\mathrm{dBCH}$ be the dual code. As is well known, thus $\mathrm{dBCH}$ is a $\left[n_{\mathrm{BCH}}, t_{\mathrm{BCH}} \log n_{\mathrm{BCH}}, \frac{n}{2}-t_{\mathrm{BCH}} \sqrt{n_{\mathrm{BCH}}}\right]_{2}$-code. Set $n_{\mathrm{BCH}}$ such that $t_{\mathrm{BCH}}\left(\log n_{\mathrm{BCH}}\right)=n$. Let enc be the encoder of $\mathrm{dBCH}$.

(11) Let Samp : $\{0,1\}^{n_{4}} \rightarrow\left(\{0,1\}^{n_{6}}\right)^{D_{s}}, n_{6}=\left(\log n_{\mathrm{BCH}}\right)$, be the sampler from Theorem 4.7 using a seeded extractor Ext $_{s}$ from Theorem 4.1 for min-entropy $n_{4} / 8$ and error $\epsilon_{\text {Samp }}=1 / 20$. Thus $D_{s}=n_{4}^{C_{s}}$ for some constant $C_{s}$.

(12) $L_{1,2}:\{0,1\}^{n} \rightarrow\{0,1\}^{n_{1}+d_{4} D^{\prime}}$ be a linear map such that $L_{1,2}(x)=v \circ y_{1,1} \circ y_{1,2} \ldots \circ y_{1, D^{\prime}} \circ y_{2}$.

(13) Let $a d v_{y}(x)=\operatorname{enc}(x)_{\mid \operatorname{Samp}\left(y_{2}\right)}$. Note that $a d v_{y}:\{0,1\}^{n} \rightarrow$ $\{0,1\}^{D_{s}}$ is a linear function. For any subset of coordinates $S \subseteq D_{S}$, let $a d v_{S}$ denote the linear map that is a projection of $a d v_{y}$ to the coordinates in $S$.

Pick a subset $S_{y}$ of size $D_{s}-\left(n_{1}+d_{4} D^{\prime}\right)$ such that the vectors in $\operatorname{con}_{a d v_{S_{y}}}$ and the vectors in $\operatorname{con}_{L_{1,2}}$ are linearly independent.

(14) We now construct a sequence of linear extractors in an iterative way similar to Step (5). Let $\mathrm{LExt}_{3}:\{0,1\}^{n} \times\{0,1\}^{d_{3}} \rightarrow$ $\{0,1\}^{n_{a d v}}, n_{a d v}=n^{10 \delta}$ be a linear seeded extractor from Theorem 4.2 set to extract from min-entropy $k / 2$ with error $2^{-\Omega\left(\sqrt{d_{3}}\right)}$. Let $D=0.9 D^{\prime}$.

(a) Let $i \leftarrow 1, \mathcal{L} \leftarrow \operatorname{con}_{L_{1,2}} \cup \operatorname{con}_{a d v_{S_{y}}}$, counter $\leftarrow 0$, rowlist $\leftarrow \emptyset$.

(b) while $i \leq D^{\prime}$ and counter $<D$ :

(i) Define the function $f_{i}(x)=\operatorname{LExt}_{3}\left(x, y_{1, i}\right)$. Note that $f_{i}:\{0,1\}^{n} \rightarrow\{0,1\}^{n a d v}$ is a linear function. If $f_{i}\left(\{0,1\}^{n}\right) \neq\{0,1\}^{n_{a d v}}$, go to (v).

(ii) Update counter $\leftarrow$ counter +1 , rowlist $\leftarrow$ rowlist $\cup\{i\}$.

(iii) For any $S \subseteq\left[n_{a d v}\right]$, let $f_{i, S}:\{0,1\}^{n} \rightarrow\{0,1\}^{|S|}$ be the projection of $f_{i}$ to the coordinates in $S$ Pick a subset $S_{i}$ of $\left[n_{a d v}\right],\left|S_{i}\right|=n_{a d v} / 2 D$ such that $\operatorname{con}_{f_{i, S_{i}}} \cup \mathcal{L}$ is a set of linearly independent vectors. Define

$\operatorname{LExt}_{3, i}(x, y)=\operatorname{LExt}_{3}(x, y)_{\mid S_{i}}$.

(iv) Update $\mathcal{L} \leftarrow \mathcal{L} \cup \operatorname{con}_{f_{i, S_{i}}}$.

(v) $i \leftarrow 1+1$

(15) If counter $<D$, output $0^{m}$.

(16) For each $i \in[D]$, define $a d v_{i}=i \circ y_{1, i} \circ y_{2} \circ a d v_{S_{y}}(x) \circ$ $\operatorname{LExt}_{3, i}\left(x, y_{1, i}\right)$. Let $\left|a d v_{i}\right|=h$.

(17) $L_{a d v}:\{0,1\}^{n} \rightarrow\{0,1\}^{n_{1}+d_{4} D+h D}$ be a linear map such that $L_{1,2}(x)=v \circ y_{1,1} \circ y_{1,2} \ldots \circ y_{1, D} \circ y_{2} \circ a d v_{1} \circ \ldots \circ a d v_{D}$.

(18) For each $i \in[D]$, let $y_{-2, i}=\operatorname{slice}\left(r_{i}, d_{4}+1, n_{3}\right)$. We iteratively pick subsets of coordinates and project these variables to these subsets in the following way:

(a) Let $i \leftarrow 1, \mathcal{L} \leftarrow \operatorname{con}_{L_{a d v}}$ :

(b) while $i \leq D$ :

(i) Define the function $f_{i}(x)=y_{-2, i}$. Note that $f_{i}:\{0,1\}^{n} \rightarrow\{0,1\}^{n_{3}-d_{4}}$ is a linear function.

(ii) Update counter $\leftarrow$ counter +1 , rowlist $\leftarrow$ rowlist $\cup\{i\}$. (iii) For any $S \subseteq\left[n_{3}-d_{4}\right]$, let $f_{i, S}:\{0,1\}^{n} \rightarrow$ $\{0,1\}^{|S|}$ be the projection of $f_{i}$ to the coordinates in $S$. Pick a subset $S_{i}$ of $\left[n_{3}-d_{4}\right],\left|S_{i}\right|=$ $\left(n_{3}-d_{4}\right) / 2$ such that $\operatorname{con}_{f, S_{i}} \cup \mathcal{L}$ is a set of linearly independent vectors.

(iv) Update $r_{i} \leftarrow y_{1} \circ y_{2} \circ\left(y_{-2, i}\right)_{\mid S_{i}}$

(v) Update $\mathcal{L} \leftarrow \mathcal{L} \cup \operatorname{con}_{f_{i, s_{i}}}$.

(vi) $i \leftarrow 1+1$

(19) Let $n_{3}^{\prime}=\left(n_{3}+d_{4}\right) / 2$.

(20) Let $d_{5}=d_{4}+D^{20} d_{4}$. For each $i \in[D]$, let $y_{3, i}=\operatorname{Slice}\left(r_{i}\right.$, $\left.d_{4}+1, d_{5}\right)$. Let $n_{5}=d_{5}-d_{4}$

(21) Let $d_{6}=d_{5}+D^{40} d_{5}$. Let $y_{4}=\operatorname{Slice}\left(r_{1}, d_{5}+1, d_{6}\right) \circ \ldots \circ$ Slice $\left(r_{D}, d_{5}+1, d_{6}\right)$. Let $n_{6}=D\left(d_{6}-d_{5}\right)$.

(22) Let ACB : $\{0,1\}^{n_{5}} \times\{0,1\}^{n_{6}} \times\{0,1\}^{h} \rightarrow\{0,1\}^{m_{1}}$ be the correlation breaker from Theorem 5.2 setup using the following components:

- $\operatorname{LExt}_{1, \mathrm{ACB}}:\{0,1\}^{n_{6}} \times\{0,1\}^{d_{7}} \rightarrow\{0,1\}^{d_{7}}$ and $\mathrm{LExt}_{2, \mathrm{ACB}}$ : $\{0,1\}^{m_{1}} \times\{0,1\}^{d_{7}} \rightarrow\{0,1\}^{d_{7}}$ be

$\left(k_{\mathrm{ACB}}=C_{2} h D^{3} \log ^{2}\left(n / \epsilon_{A C B}\right), \epsilon_{\mathrm{ACB}}=2^{-4 d_{4} D}\right)$-strong

linear seeded extractors, $m_{1}=k_{\mathrm{ACB}}+C_{2} D d_{7}+4 d_{4} D$ instantiated from Theorem 4.2, where $C_{2}$ is a large enough constant.

- $\operatorname{LExt}_{3, \mathrm{ACB}}:\{0,1\}^{n_{5}} \times\{0,1\}^{d_{7}} \rightarrow\{0,1\}^{m_{1}}$ be a $\left(k_{2, \mathrm{ACB}}=\right.$ $\left.\left(n_{2}^{\prime}\right)^{1.1}, \epsilon_{\mathrm{ACB}}\right)$-strong linear seeded extractor.

(23) For each $i \in[D]$, let

$$
w_{i}=\operatorname{ACB}\left(y_{3, i}, y_{4}, a d v_{i}\right) .
$$

(24) For each $i \in[D]$, let $y_{5, i}=\operatorname{Slice}\left(r_{i}, d_{6}+1, n_{3}^{\prime}\right)$. Let $n_{7}=$ $\left(n_{3}^{\prime}-d_{6}\right)$.

(25) Let iExt : $\{0,1\}^{n_{7}} \times\{0,1\}^{m_{1}} \rightarrow\{0,1\}^{m}, m=\Omega\left(m_{1}\right)$, be the linear seeded extractor from Theorem 4.3 set to extract from min-entropy $0.9 n_{7}$ with error $2^{-\Omega\left(m_{1}\right)}$.

(26) Let

(27) Output

$$
y_{6, i}=\mathrm{iExt}\left(y_{5, i}, w_{i}\right)
$$

$$
z=\bigoplus_{i=1}^{D} y_{6, i} .
$$

THeOREm 8.1. There exists a constant $C$ such that for all $n, k, w>0$ and any $\delta>0$ with $w<n^{\beta}, \beta=\delta /(3 C)$ and $k \geq n^{C \delta}$, there exists an efficient function anmExt : $\{0,1\}^{n} \rightarrow\{0,1\}^{m}$ such that if $\mathrm{X}$ is a $w$-affine source with min-entropy at least $k$ and $\mathcal{A}:\{0,1\}^{n} \rightarrow\{0,1\}^{n}$ is an affine function with no fixed points, then

$$
\left|\operatorname{ianmExt}(\mathbf{X}), \operatorname{anmExt}(\mathcal{A}(\mathbf{X}))-\mathbf{U}_{m}, \operatorname{ianmExt}(\mathcal{A}(\mathbf{X}))\right| \leq 2^{-n^{\Omega(1)}}
$$

\subsection{The Sampling Algorithm}

We use the variable names in algorithm for ianmExt in Section 8.1 to describe our sampling algorithm. We use the following simple algorithm that takes as input $z \in\{0,1\}^{m}$ :

(1) Sample $v$ from $\mathbf{U}_{n_{1}}$. For each $i \in[D]$, sample $y_{1, i}$ from $\mathbf{U}_{d_{3}}$. Sample $y_{2}$ from $U_{n_{4}}$. For each $i \in[D]$, generate the remaining bits of $a d v_{i}$ uniformly.

(2) For each $i \in[D]$, sample $y_{3, i}$ from $\mathbf{U}_{n_{5}}$. Sample $y_{4}$ from $U_{n_{6}}$. 
(3) Using the variables samples, and the algorithm for ianmExt in Section 8.1, for each $i \in[D]$ compute the variable $w_{i}=$ $\operatorname{ACB}\left(y_{3,1}, y_{4}, a d v_{i}\right)$.

(4) For each $i \in[D-1]$, generate independent uniform strings $y_{6, i} \in\{0,1\}^{m}$. Set $y_{6, D}=\left(\bigoplus_{i=1}^{D-1} y_{6, i}\right) \oplus z$.

(5) For each $i \in[D]$, uniformly sample the variable $y_{5, i}$ from the space of all solutions of the equation $\operatorname{iExt}\left(., w_{i}\right)=y_{6, i}$

(6) Note that all the random variables sampled so far are linear functions in $x$, and hence places linear constraints on $x$. Sample $x$ uniformly from the largest subspace in $\{0,1\}^{n}$ that satisfies all these linear constraints.

We observe that all the steps of the above algorithm can be executed in time poly $(n)$ with access to random bits. This follows from the fact that in each step, we are either sampling uniform bits, or sampling uniformly from a subspace with access to a basis. We refer the reader to the full version for a proof of correctness.

\section{ACKNOWLEDGMENTS}

The authors would like to thank the anonymous referees for their valuable comments and helpful suggestions. We also thank Pratyay Mukherjee for helpful comments. This research was partly done when Eshan Chattopadhyay was a student in UT Austin. His research was partially supported by NSF Grants CCF-1412958, CCF1526952, and the Simons Collaboration on Algorithms and Geometry. Xin Li's research was partially supported by NSF Grant CCF-1617713.

\section{REFERENCES}

[1] D. Aggarwal, Y. Dodis, T. Kazana, and M. Obremski. 2015. Non-malleable Reductions and Applications. (2015). To appear in STOC.

[2] Divesh Aggarwal, Yevgeniy Dodis, and Shachar Lovett. 2014. Non-malleable Codes from Additive Combinatorics. In STOC.

[3] Shashank Agrawal, Divya Gupta, Hemanta K. Maji, Omkant Pandey, and Manoj Prabhakaran. 2015. A Rate-Optimizing Compiler for Non-malleable Codes Against Bit-Wise Tampering and Permutations. In Theory of Cryptography 12th Theory of Cryptography Conference, TCC 2015, Warsaw, Poland, March 23-25, 2015, Proceedings, Part I. 375-397.

[4] Noga Alon, Jehoshua Bruck, Joseph Naor, Moni Naor, and Ron M. Roth. 1992 Construction of asymptotically good low-rate error-correcting codes through pseudo-random graphs. IEEE Transactions on Information Theory 38 (1992), 509-516.

[5] Marshall Ball, Dana Dachman-Soled, Mukul Kulkarni, and Tal Malkin. 2016 Non-Malleable Codes for Bounded Depth, Bounded Fan-in Circuits. In TCC.

[6] Jean Bourgain. 2007. On the Construction of Affine Extractors. GAFA Geometric And Functional Analysis 17, 1 (2007), 33-57. DOI : https://doi.org/10.1007/ s00039-007-0593-z

[7] Eshan Chattopadhyay, Vipul Goyal, and Xin Li. 2016. Non-Malleable Extractors and Codes, with their Many Tampered Extensions. In STOC.

[8] Eshan Chattopadhyay and Xin Li. 2016. Explicit Non-Malleable Extractors, Multi-Source Extractors and Almost Optimal Privacy Amplification Protocols. Electronic Colloquium on Computational Complexity (ECCC) (2016). http://eccc. hpi-web.de/report/2016/036

[9] Eshan Chattopadhyay and Xin Li. 2016. Extractors for Sumset Sources. In STOC.

[10] Eshan Chattopadhyay and David Zuckerman. 2014. Non-malleable Codes against Constant Split-State Tampering. In Proceedings of the 55th Annual IEEE Symposium on Foundations of Computer Science. 306-315.
[11] Eshan Chattopadhyay and David Zuckerman. 2016. Explicit Two-Source Extractors and Resilient Functions. In STOC.

[12] Mahdi Cheraghchi and Venkatesan Guruswami. 2014. Capacity of non-malleable codes. In ITCS. 155-168.

[13] Mahdi Cheraghchi and Venkatesan Guruswami. 2014. Non-malleable Coding against Bit-Wise and Split-State Tampering. In TCC. 440-464.

[14] Benny Chor and Oded Goldreich. 1988. Unbiased Bits from Sources of Weak Randomness and Probabilistic Communication Complexity. 17, 2 (1988), 230-261.

[15] Gil Cohen. 2015. Local Correlation Breakers and Applications to Three-Source Extractors and Mergers. In Proceedings of the 56th Annual IEEE Symposium on Foundations of Computer Science.

[16] Gil Cohen. 2016. Making the Most of Advice: New Correlation Breakers and Their Applications. Electronic Colloquium on Computational Complexity (ECCC) (2016). http://eccc.hpi-web.de/report/2016/052

[17] Gil Cohen. 2016. Non-Malleable Extractors - New Tools and Improved Constructions. In CCC.

[18] Ronald Cramer, Yevgeniy Dodis, Serge Fehr, Carles Padró, and Daniel Wichs. 2008. Detection of Algebraic Manipulation with Applications to Robust Secret Sharing and Fuzzy Extractors. In EUROCRYPT. 471-488.

[19] Yevgeniy Dodis and Daniel Wichs. 2009. Non-malleable extractors and symmetric key cryptography from weak secrets. In STOC. 601-610.

[20] Stefan Dziembowski, Tomasz Kazana, and Maciej Obremski. 2013. Non-malleable Codes from Two-Source Extractors. In CRYPTO (2). 239-257.

[21] Stefan Dziembowski and Krzysztof Pietrzak. 2007. Intrusion-Resilient Secret Sharing. In Proceedings of the 48th Annual IEEE Symposium on Foundations of Computer Science (FOCS '07). IEEE Computer Society, Washington, DC, USA, 227-237. DOI : https://doi.org/10.1109/FOCS.2007.35

[22] Stefan Dziembowski, Krzysztof Pietrzak, and Daniel Wichs. 2010. Non-Malleable Codes. In ICS. 434-452.

[23] Sebastian Faust, Pratyay Mukherjee, Daniele Venturi, and Daniel Wichs. 2014. Efficient Non-malleable Codes and Key-Derivation for Poly-size Tampering Circuits. Springer Berlin Heidelberg, Berlin, Heidelberg, 111-128. DOI : https://doi.org/10. 1007/978-3-642-55220-5_7

[24] Venkatesan Guruswami, Christopher Umans, and Salil P. Vadhan. 2009. Unbalanced expanders and randomness extractors from Parvaresh-Vardy codes. $\mathcal{F}$. ACM 56, 4 (2009).

[25] Johan Hastad. 1987. Computational Limitations of Small-depth Circuits. MIT Press, Cambridge, MA, USA.

[26] Xin Li. 2013. Extractors for a Constant Number of Independent Sources with Polylogarithmic Min-Entropy. In Proceedings of the 54th Annual IEEE Symposium on Foundations of Computer Science. 100-109.

[27] Xin Li. 2015. Improved Two-Source Extractors, and Affine Extractors for Polylogarithmic Entropy. Technical Report TR15-125. ECCC.

[28] Xin Li. 2015. Three-Source Extractors for Polylogarithmic Min-Entropy. In Proceedings of the 56th Annual IEEE Symposium on Foundations of Computer Science.

[29] Xin Li. 2016. Improved non-malleable extractors, non-malleable codes and independent source extractors. arXiv preprint arXiv:1608.00127 (2016).

[30] Anup Rao. 2009. Extractors for Low-Weight Affine Sources. In Proceedings of the 24th Annual IEEE Conference on Computational Complexity.

[31] Ran Raz, Omer Reingold, and Salil Vadhan. 2002. Extracting all the Randomness and Reducing the Error in Trevisan's Extractors. FCSS 65, 1 (2002), 97-128.

[32] Luca Trevisan. 2001. Extractors and Pseudorandom Generators. (2001), 860-879.

[33] Emanuele Viola. 2011. Extractors for Circuit Sources. In IEEE 52nd Annual Symposium on Foundations of Computer Science, FOCS 2011, Palm Springs, CA, USA, October 22-25, 2011. 220-229.

[34] Emanuele Viola. 2014. Extractors for Circuit Sources. SIAM 7. Comput. 43, 2 (2014), 655-672. DOI : https://doi.org/10.1137/11085983X

[35] D. Zuckerman. 1990. General weak random sources. 2013 IEEE 54th Annual Symposium on Foundations of Computer Science 0 (1990), 534-543 vol.2. DOI : https://doi.org/10.1109/FSCS.1990.89574

[36] David Zuckerman. 1997. Randomness-Optimal Oblivious Sampling. Random Structures and Algorithms 11 (1997), 345-367.

[37] David Zuckerman. 2007. Linear Degree Extractors and the Inapproximability of Max Clique and Chromatic Number. Theory of Computing (2007), 103-128. 